\title{
Silicon Nanoscale Materials: From Theoretical Simulations to Photonic Applications
}

\author{
Leonid Khriachtchev, ${ }^{1}$ Stefano Ossicini, ${ }^{2}$ Fabio Iacona, ${ }^{3}$ and Fabrice Gourbilleau ${ }^{4}$ \\ ${ }^{1}$ Department of Chemistry, University of Helsinki, P.O. Box 55, 00014 Helsinki, Finland \\ ${ }^{2}$ Dipartimento di Scienze e Metodi dell'Ingegneria, Università di Modena e Reggio Emilia, Via Amendola, 2 Pad. Morselli, \\ 42122 Reggio nell'Emilia, Italy \\ ${ }^{3}$ MATIS IMM CNR, Via Santa Sofia 64, 95123 Catania, Italy \\ ${ }^{4}$ CIMAP, CNRS/CEA/ENSICAEN/UCBN, 6 boulevard Maréchal Juin, 14050 Caen Cedex 4, France
}

Correspondence should be addressed to Leonid Khriachtchev, leonid.khriachtchev@helsinki.fi

Received 18 December 2011; Revised 13 March 2012; Accepted 13 March 2012

Academic Editor: David Lee Phillips

Copyright (C) 2012 Leonid Khriachtchev et al. This is an open access article distributed under the Creative Commons Attribution License, which permits unrestricted use, distribution, and reproduction in any medium, provided the original work is properly cited.

\begin{abstract}
The combination of photonics and silicon technology is a great challenge because of the potentiality of coupling electronics and optical functions on a single chip. Silicon nanocrystals are promising in various areas of photonics especially for light-emitting functionality and for photovoltaic cells. This review describes the recent achievements and remaining challenges of Si photonics with emphasis on the perspectives of Si nanoscale materials. Many of the results and properties can be simulated and understood based on theoretical studies. However, some of the key questions like the light-emitting mechanism are subjects of intense debates despite a remarkable progress in the recent years. Even more complex and important is to move the known experimental observations towards practical applications. The demonstrated devices and approaches are often too complex and/or have too low efficiency. However, the challenge to combine optical and electrical functions on a chip is very strong, and we expect more research activity in the field of Si nanophotonics in the future.
\end{abstract}

\section{Introduction}

Photonics is a key technology of this century. The fast development of optical communications demonstrates the advantages of photonics with respect to electrical signalling. As the size of devices scales down, photonics becomes even more attractive because the resistance and capacitance of metal interconnects increase, and optical interconnects have many advantages. Silicon is the basis material in electronics. Thus, the combination of photonics with silicon technology is a great current challenge because of the potentiality of coupling electronics and optical functions (light emission, amplification, modulation, waveguiding, optical memory, etc.) on a single chip. Silicon nanocrystals (Si-nc) are promising in various areas of photonics especially for lightemitting functionality and, more recently, for applications in advanced photovoltaic cells [1-5]. World market for Si photonics is expected to be huge; however, many fundamental and practical problems should be solved in order to develop this technology.

Silicon-based light-emitting device with high efficiency is a missing part in the design of complete optoelectronic circuits based on silicon technology. Silicon is a bad light emitter due to the indirect nature of the band gap. It was about two decades ago when bright emission from porous silicon was discovered, and it was connected with the nanocrystalline structure [6]. This discovery seemed to remove the main obstacle to silicon photonics, and the number of research papers in this area increased enormously [7]. It has been shown that the band gap is a function of the crystalline size if the spatial extend of the electron wave function becomes comparable with the crystallite diameter (photon confinement model). Studies of Si-nc embedded in silica have been stimulated by reports on optical gain in this material $[8,9]$. 
The unusual properties of Si-nc embedded in silica are tightly connected to the stable passivation ensured by the matrix, which eliminates dangling bonds through the formation of $\mathrm{Si}-\mathrm{O}$ single bonds and $\mathrm{Si}=\mathrm{O}$ double bonds [10] and constitutes a low defect density medium where nonradiative recombination processes are strongly reduced. Unfortunately, silica is also a very good insulating material so that the carrier injection through an $\mathrm{SiO}_{2}$ layer containing $\mathrm{Si}$ $\mathrm{nc}$ is difficult. As a consequence, this material, in spite of its appealing optical properties, has been considered for a long time poorly useful for the realization of electrically pumped light sources. Despite this drawback, several prototypes of light-emitting devices based on Si-nc have been proposed. Well before Canham's discovery of the light-emitting properties of porous silicon, the electroluminescence (EL) of small $\mathrm{Si}$ clusters embedded in $\mathrm{SiO}_{2}$ matrix was reported in the pioneering work of DiMaria et al. [11]. In the following years, the above-discussed difficulties concerning the electrical excitation of Si-nc have been overcome by confining them in ultrathin layers and/or by properly tuning their size and density; these strategies allowed the fabrication of devices exhibiting room temperature EL in the visible and near infrared region at reasonably low voltages [12-16]. The demonstration that the cross-section for electrical excitation of Si-nc is about two orders of magnitude higher than that observed under optical pumping has suggested that Si-nc may have important perspectives for practical applications [17].

An alternative approach that has been used to efficiently produce photons from Si-based materials exploits the introduction of light-emitting impurities, such as Er. Er ions emit at $1.54 \mu \mathrm{m}$, which is a strategic wavelength for telecommunication, because it corresponds to a minimum in the loss spectrum of silica optical fibers. Room temperature operating diodes were fabricated based on Erdoped crystalline silicon with an internal quantum efficiency of $0.05 \%$ under reverse bias $[18,19]$. More recently, Erdoped Si nanoclusters embedded in silica have attracted the interest of the scientific community as a promising light source at $1.54 \mu \mathrm{m}$ [20-26]. In fact, $e-h$ pairs photoexcited in Si nanoclusters can decay nonradiatively by transferring their energy to a nearby Er ion which, in turn, decays by emitting a $1.54-\mu \mathrm{m}$ photon. In this case, the luminescence intensities two orders of magnitude higher than in Er-doped silica have been measured at room temperature [21], as a result of an increased Er excitation cross-section with respect to direct photon absorption [25]. This sensitization effect stimulated the fabrication of the EL devices based on Er-doped Si nanostructures; their intense and stable room temperature EL emission at $1.54 \mu \mathrm{m}$ is the result of the energy-transfer process from electrically excited nanoclusters to neighboring ions [27-30].

Another important task of Si nanostructures is (in opposite to EL) to generate electrical energy from solar light taking benefit of the monitoring of the Si bandgap through the Si-nc size. This is one of the concepts of the third generation of photovoltaic cells with the final objective of achieving an all-Si tandem solar cell [31]. Such a structure offers the opportunity to overcome the well-known process of thermalization in the cell, which represents one of the major sources of loss occurring in the Si-based photovoltaic module.

Control of optical properties of materials is central in designing photonic components (light emitters, optical waveguides, optical memory, etc.). In order to develop the advanced materials suitable for photonic devices, one should prepare their properties in a predictable and repeatable way and control the self-assembly at the nanoscale. Programming of the material properties is crucial not only at the stage of sample fabrication but also for postdeposition treatments, which can be irradiation by light and energetic particles and the effect of voltage. An example of such an effect is reversible laser-controlled stress of Si-nc in silica [32]. It is not possible to understand and develop the optical properties of nanoparticles without fundamental information on their microscopic structure.

A large number of theoretical works, based on empirical and on ab initio approaches, are now available (see, e.g., $[1,3,33])$. The indisputable superiority of the first-principles approach is gloomed by its applicability to systems of less than a thousand atoms. Computationally more feasible techniques that can handle more than 10000 atoms exist. The most common ones are the envelope function $\mathrm{k} \cdot \mathrm{p}$ [34], the semiempirical tight-binding [33], and the semiempirical pseudopotential techniques [35]. The envelope function approach lacks the atomistic touch and presents qualitative and quantitative errors [36]. The tight-binding approach has been employed by several groups [33]. The pseudopotential method, in particular, in the version that uses a basis set formed by the linear combination of bulk bands of the constituent of the nanostructure [37], has been used in the context of Si-nc for studying the effects of nanocrystals aggregation, the linear and nonlinear optical properties, the Auger recombination, and the carrier multiplication [3841]. Regarding first-principles approaches, a large majority of the studies have used the density-functional theory (DFT) in the local density approximation (LDA) [42].

The main objective of this review is to describe the recent achievements and remaining challenges of $\mathrm{Si}$ photonics with an emphasis on the perspectives of $\mathrm{Si}$ nanoscale materials. Some attention is paid to the preparation and analytical methods used in the field. We will describe the subjects, which are familiar to us in our practical work, from theoretical and fundamental results to practical applications. Although we try to mention the most important research directions in the field, many efforts and ideas are not described. The reason for this is the limited space and the huge volume of the published results. More information can be found in numerous books in the field, for example, in [1-5].

\section{Theoretical Simulations of Oxidized and Embedded Si Nanostructures}

Despite the remarkable progress in theoretical simulations of the optical properties of Si-based materials, an exhaustive understanding is still lacking. The reason is twofold, on one 
side the empirical parameters are not so easy transferable to nanoscale systems, and on the other side the ab initio DFTLDA suffers of deficiency in the correct evaluation of the excitation energies. Thus, the inclusion of many-body effects is mandatory in the theoretical description of the excitedstate properties. In particular, the quasiparticle structure is a key for the calculation of the electronic gap as the inclusion of excitonic effects is necessary for a correct description of the optical properties [43]. The obtained results indicate that the light-emission mechanism in oxidized Si-nc seems to be more complex than that explained by the photon confinement model and that both a strong interplay between the silicon nanocrystal and the surrounding host environment and an active role of the interface regions must be taken into account. Several theoretical calculations have been applied to investigate the possible active role of the interface on the optoelectronic properties of Si-nc. Most of these calculations address mainly the problem of absorption, but little has been done concerning the excited-state configurations. The understanding of the physical mechanisms at the origin of phenomena like the Stokes shift between absorption and emission or the optical gain implies an analysis of the optically excited nanostructures.

2.1. Ab Initio Methods. The electronic and optical properties of complex systems like oxidized and embedded Si-nc can be now studied within $a b$ initio methods, thanks to the impressive developments of theoretical approaches and computer power. Within the Born-Oppenheimer approximation to decouple the ionic and electronic dynamic, the equation that governs the properties of a system is a many-body equation, whose direct solution is, however, a formidable task. It is hence necessary to resort to approximations. In particular, the static density functional theory [44] avoids dealing directly with many bodies by mapping the interacting system into a fictitious noninteracting system, which is then described by single particle Kohn-Sham equations [45]. This method, in particular, in the so-called local density approximation (LDA), has been very successful in the last decades regarding the calculation of ground state properties. However, DFT-LDA severely underestimated the band gaps for semiconductors and insulators. Thus, the inclusion of many-body effects is mandatory for a correct description of the electronic band gap and of the optical spectra.

The Green function approach maps the many-body electronic problem to a system of quasiparticles which describe the excitations in terms of a particle finite lifetime that represent the extra electron, and/or the extra hole, added to the system plus its screened interaction with the electrons of the system. Thus, a successful correction to the electronic band gap of semiconductor and insulator is obtained by calculating the separate electron and hole quasiparticles energies via the self-energy approximation in the so-called GW method [46].

The knowledge of the quasiparticles energies, however, is still not sufficient to describe correctly the optical properties due to the fact that the optical spectra are related to the creation of electron-hole pairs. The interaction between the positively and negatively charged quasiparticles can, in fact, lead to a strong shift of the peak positions as well as to distortions of the spectral line shape. Within the manybody perturbation theory (MBPT) framework [47], such interaction is taken into account by the solution of the Bethe-Salpeter equation for the polarizability, considering the so-called excitonic Hamiltonian. An alternative approach is represented by the time-dependent DFT (TDDFT) [48]. TDDFT is expected to be more efficient than MBPT; however, it has been recently demonstrated that in the case of Si-nc TDDFT does not take into account correctly the screened Coulomb interaction [49]. Indeed, in order to compute correct optical spectra, it is necessary to include local field effects that are important for inhomogeneous systems, like Si-nc, where the dielectric function changes abruptly at the interfaces.

To summarize, in order to compute the excitonic spectra, one needs a well-converged structure calculation, KohnSham wave functions, quasiparticle energies within GW, local field effects, and the screened Coulomb interactions present in the excitonic Hamiltonian.

2.2. Oxidized Si-nc. The experimental data show that the exposition of Si-nc to oxygen produces an impact on their optical properties. Thus, the oxidation process at the surface of Si-nc has to be taken into account in the calculations. Wolkin et al. [10] have observed that oxidation introduced defects in the Si-nc band gap which pin the transition energy. They and other authors $[50,51]$ suggest that the formation of $\mathrm{Si}=\mathrm{O}$ double bonds is responsible for the red shift of the optical absorption edge upon oxidation. Gatti and Onida [52] considered small oxidized Si clusters and found similar results, whereas Vasiliev et al. [53] have pointed out that similar results can be obtained also for an $\mathrm{O}$ atom connecting two $\mathrm{Si}$ atoms (bridge bond) at the nanocrystal surface.

Detailed results related to the effect of oxidation process for Si-nc on the structural and optical properties have been reported by Luppi et al. [54]. They have considered small Si-nc of different size terminated by hydrogen, and then two types of bonds between $\mathrm{Si}$ and $\mathrm{O}$ atoms have been introduced at the nanocrystal surface: the $\mathrm{Si}-\mathrm{O}-\mathrm{Si}$ bridge bond (indicated with $\mathrm{Si}>\mathrm{O}$ in Figure 1 ) and the $\mathrm{Si}=\mathrm{O}$ double bond. In all cases, full relaxation with respect to the atomic positions within DFT has been performed both in the ground and excited state configuration. The results show that the bridge bonding leads to the more stable isomer configuration, even if the double bonded one is not so far in energy. Moreover, in going from the ground to the excited state, the promotion of an electron leads to a distortion of the initial cluster geometry that depends on the surface termination. For the double bond case, the distortion is mainly localized near the $\mathrm{O}$ atoms, whereas in the bridge structure the deformation is localized around the $\mathrm{Si}-\mathrm{O}-\mathrm{Si}$ bond determining a strain in the $\mathrm{Si}-\mathrm{Si}$ atomic distances. To allow a direct comparison with the experimental data, both the absorption and the emission optical spectra have been calculated. The optical response has been evaluated for the calculated ground and excited state geometries including the self-energy corrections by means of the GW method and the excitonic effects through the solution of the Bethe-Salpeter 


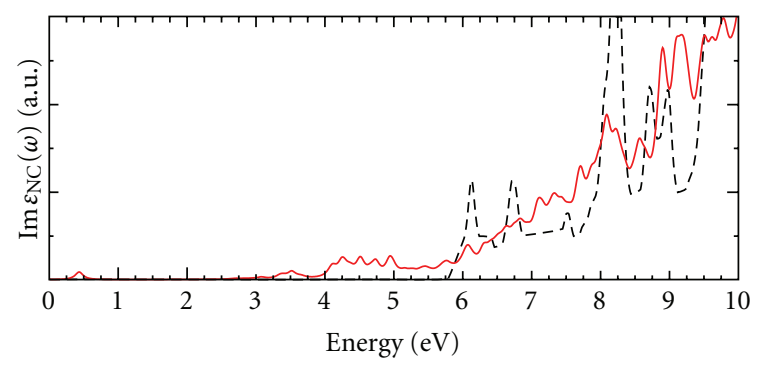

- - $\mathrm{Si}_{10} \mathrm{H}_{16}$ absorption

- $\mathrm{Si}_{10} \mathrm{H}_{16}$ emission

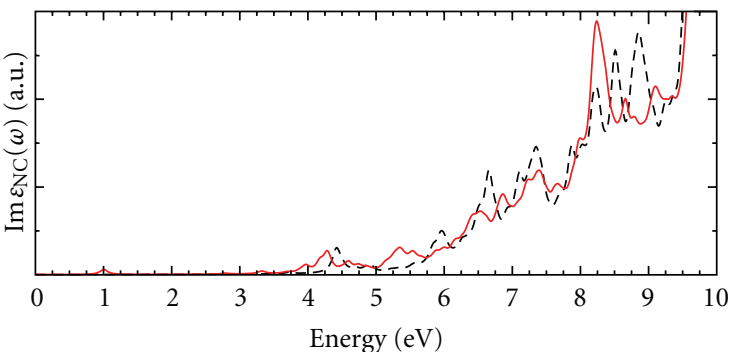

- - $\mathrm{Si}_{10} \mathrm{H}_{14}=\mathrm{O}$ absorption

- $\mathrm{Si}_{10} \mathrm{H}_{14}=\mathrm{O}$ emission
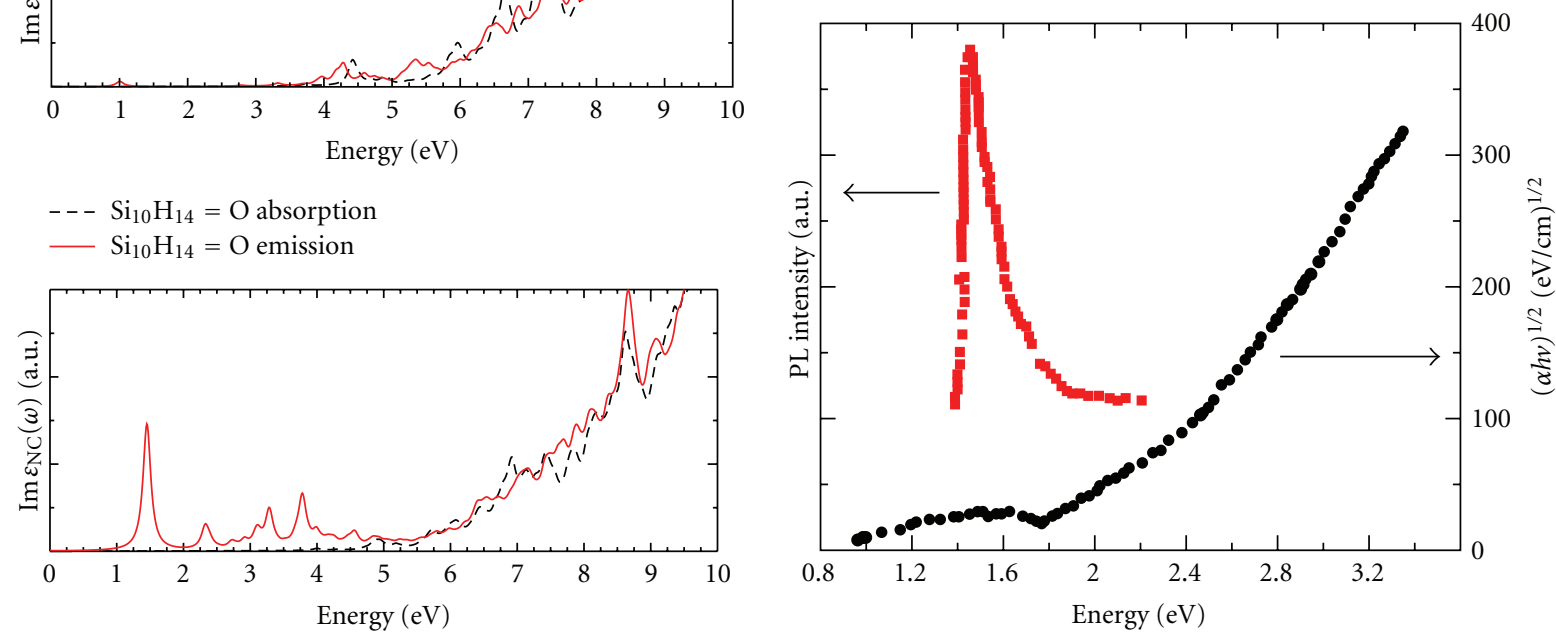

- - $\mathrm{Si}_{10} \mathrm{H}_{14}>\mathrm{O}$ absorption

- $\mathrm{Si}_{10} \mathrm{H}_{14}>\mathrm{O}$ emission

(a)

(b)

FIGURE 1: (a) Calculated absorption (dashed line) and emission (solid line) spectra: (from top to bottom) imaginary part of the dielectric function for the $\mathrm{Si}_{10} \mathrm{H}_{16}$ nanocrystal, the $\mathrm{Si}_{10} \mathrm{H}_{14}=\mathrm{O}$ nanocrystal with a double bonded oxygen, and the $\mathrm{Si}_{10} \mathrm{H}_{14}>\mathrm{O}$ nanocrystal with a bridge bonded oxygen [54]. (b) Experimental results for absorption (black curve on the right) and emission (red curve on the left) by Ma et al. [55].

equation. Also the effect of the local field has been included to take into account the inhomogeneity of the nanocrystals.

The main result for both absorption and emission geometry is the opening of the LDA band gap with the GW corrections, which is weakly dependent on the nanocrystal termination and much larger than the corresponding value in bulk silicon. Concerning the Bethe-Salpeter and local field effects, they determine a sort of compensation of the self-energy and excitonic contribution. A clearer insight in the results is given in Figure 1(a) that shows the calculated absorption and emission spectra for the oxidized $\mathrm{Si}_{10}$-based nanocrystals; moreover, these spectra are compared with those of the fully hydrogenated cluster. Concerning the absorption spectra, all the cases show a similar smooth increase in the absorption features.
The situation is dramatically different for the emissionrelated spectra. Only in the case of the $\mathrm{Si}-\mathrm{O}-\mathrm{Si}$ bridge bond, an important excitonic peak, well separated from the rest of the spectrum, is present at energy of about $1.5 \mathrm{eV}$. Bound excitons are also present in the other two cases, but the related transitions are almost dark, and the emission intensity is very low. If one compares the theoretical results with the experimental spectra by Ma et al. [55] (Figure1(b)) the conclusion is evident. The presence of a $\mathrm{Si}-\mathrm{O}-\mathrm{Si}$ bridge bond at the surface of the nanocrystal and the relative deformation localized around the bond can explain the nature of luminescence in the Si-nc; only in this case the presence of a clear excitonic peak in the emission spectra, redshifted with respect to the absorption onset, provides an explanation for the observed Stokes shift between absorption 


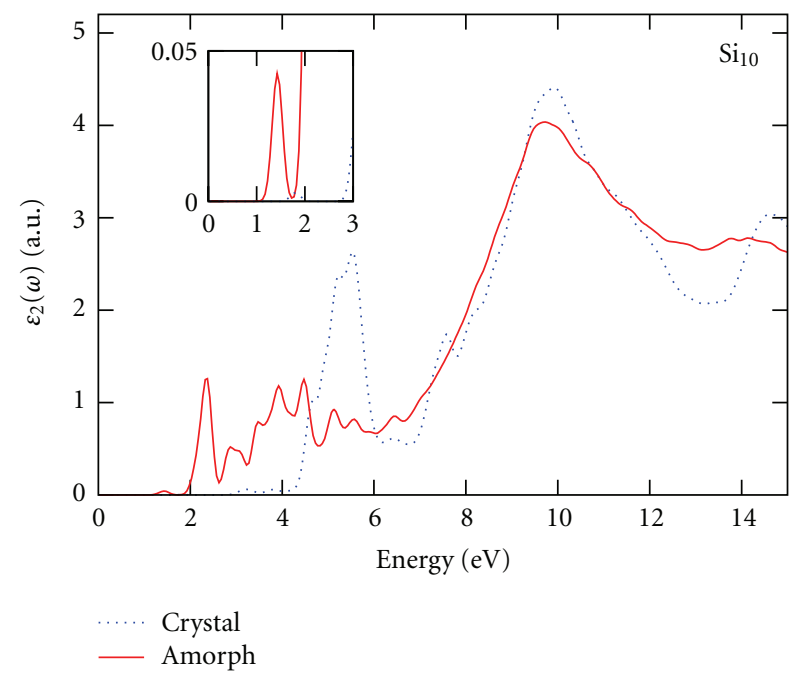

(a)

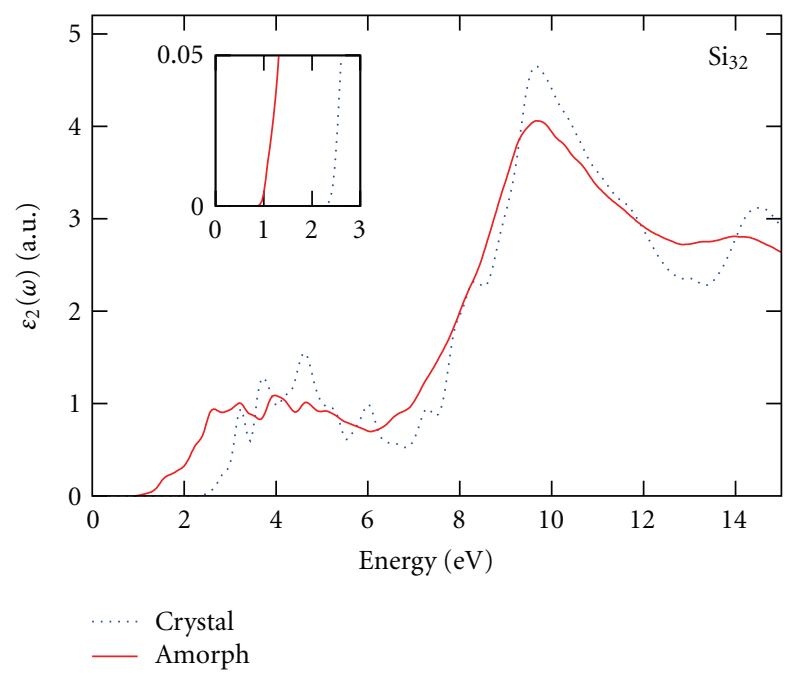

(b)

FIguRE 2: Imaginary part of the dielectric function at DFT-RPA level for the crystalline (dotted) and amorphous (solid) $\mathrm{Si}_{10}$ and $\mathrm{Si}_{32}$ embedded Si-nc [58]. The insets show an enlargement of the spectra at low energies. The spectra are per unit volume.

and emission and for the red PL. Similar results have been obtained for nanocrystals of larger size [54].

2.3. Si-nc Embedded in a $\mathrm{SiO}_{2}$ Matrix. The calculation of the optical properties of silicon nanocrystals embedded in a silica matrix is a formidable task due to the huge number of atoms in the unit cell and the crystalline or amorphous nature of the systems. The first attempts were published in 2003 [56, 57] taking into account very small Si-nc embedded in beta-cristobalite (BC) $\mathrm{SiO}_{2}$, that is well known to give rise to one of the simplest $\mathrm{Si} / \mathrm{SiO}_{2}$ interfaces. The main result for the electronic properties was the presence, at the valence and conduction band edges, of interface-related states that originate strong absorption features in the optical region.

In a series of recent articles, Guerra et al. have considered Si-nc of different sizes embedded in a crystalline or amorphous $\mathrm{SiO}_{2}$ matrix [58-62]. The crystalline-embedded structures have been obtained from a BC cubic matrix by removing all the oxygen atoms included in a cutoff sphere, whose radius determines the size of the nanocrystal. No dangling bonds are present at the Si-nc surface, and all the $\mathrm{O}$ atoms at the $\mathrm{Si} / \mathrm{SiO}_{2}$ interface are single bonded with the $\mathrm{Si}$ atoms of the Si-nc. The optimized structure has been achieved by relaxing the total volume of the cell. Up to 648 atoms have been included in the cell, and nanocrystals up to a diameter of $1 \mathrm{~nm}$ have been considered. After the relaxation, the silica matrix appears strongly distorted near the Si-nc, and also the nanocrystal shows a strained structure with respect to bulk silicon. Similar results have been obtained by other authors [63-65]. For the amorphousembedded structures, the glass model has been generated using molecular dynamic simulations of quenching from a melt. Amorphous Si nanoclusters of different sizes have been obtained starting from a $\mathrm{Si}_{64} \mathrm{O}_{128}$ or from a $\mathrm{Si}_{216} \mathrm{O}_{432}$ glass. It has been found that the number of $\mathrm{Si}-\mathrm{O}-\mathrm{Si}$ bridge bonds increases with the dimension of the nanocrystal in agreement with other structures obtained by different methods [6669]. In all cases, the results have been discussed in terms of crystallinity versus amorphization, size, degree of oxidation, and strain.

The effects induced by the amorphization process and by the size on the optical properties of the embedded $\mathrm{Si}$ nanoclusters are depicted in Figure 2, where the calculated absorption spectra for both crystalline and amorphous phase are reported for nanoclusters of two different sizes [58]. One notes that the amorphization produces a net change in the shape of the spectrum for the smaller clusters, whereas in the larger one there is only a red shift. More important, a comparison of the experimental results for small Si-nc [70] with the calculated spectrum for $\mathrm{Si}_{32}$ shows a nice agreement in the 2 to $6 \mathrm{eV}$ region.

Concerning strain and oxidation, Guerra et al. [60] found evidences that the amount of strain exerted on the nanocrystal is connected to the interface configuration, in particular, to the number of oxygen atoms per interface silicon atoms. Moreover, while the quantum confinement dominates in the hydrogenated nanocrystals of all sizes, the behavior of hydroxided and embedded nanocrystals shows a strong correlation between the final value of the electronic gap and the oxidation degree $\Omega$ (i.e., the ratio between the number of oxygen atoms and the number of Si atoms bonded to them at the nanocrystal's surface) that seems to override the effects of quantum confinement for nanocrystals of diameters below $2 \mathrm{~nm}$ (see Figure 3 ). A competition occurs between the oxidation and the strain, in which the former tends to blueshift the absorption spectra, and the latter to redshift it. Finally, the gap of small embedded Si-nc shows an oscillating behavior with size, not strictly following the quantum confinement rule. These strong fluctuations of the electronic gap with the nanocrystal size could be 
responsible for the large broadening of the PL spectra that have been observed experimentally, also at low temperatures [71-74].

In order to investigate the best conditions for optical emission, the radiative recombination rates at different temperatures for different classes of Si-nc $(\mathrm{H}-$ and $\mathrm{OH}-$ terminated as well as $\mathrm{Si}-\mathrm{nc}$ embedded in a $\mathrm{SiO}_{2}$ matrix) in the diameter range of $0.2-1.5 \mathrm{~nm}$ were evaluated [62]. The calculated recombination rates of hydrogenated nanocrystals follow the quantum confinement feature, in which the nanocrystal diameter is the principal quantity in determining the system response. Interestingly, a completely different behavior emerges from the $\mathrm{OH}$-terminated or $\mathrm{SiO}_{2}$-embedded nanocrystals, where the number of oxygen atoms at the interface seems intimately connected to the recombination rates, thus, resulting the most important quantity for the characterization of the optical yield in such systems [75]. In particular the analysis of the results for the embedded nanoclusters reveals a clear picture in which the smallest, highly oxidized, crystalline clusters belong to the class of the most optically active $\mathrm{Si} / \mathrm{SiO}_{2}$ structures, in agreement with experimental observations [76-78].

Concerning the optical gain spectra in Si-nc, very little has been done theoretically; nevertheless, recent results for these spectra [43] show another time the importance of the electronic states located at the $\mathrm{Si} / \mathrm{SiO}_{2}$ interface.

\section{Fabrication Procedures and Characterization Methods}

Since the discovery of efficient visible photoluminescence at room temperature from porous silicon in the $90 \mathrm{~s}[6$, 7], numerous studies dealing with silicon nanostructures have been carried out, and consequently a huge effort has been devoted to the development of fabrication process and methods to characterize the prepared structures.

\subsection{Fabrication Procedures and Available Structures}

3.1.1. Deposition Methods. A variety of fabrication procedures provide highly luminescent thin films or free Si nanoparticles. For the former, the most used fabrication techniques are the plasma-enhanced chemical vapor deposition (PECVD) [79], sputtering [80, 81], ion implantation $[82,83]$, electron gun [84], and molecular beam deposition (MBD) $[85,86]$. For the latter, laser pyrolysis is a powerful approach to produce nanopowder having a slight size dispersion [87]. The importance of the control of the size in the quantum confinement process has led the grower community to develop new structures and/or processes that allow to reach such an issue. Among all the approaches reported in the literature, one of the most promising concerns the multilayer approach in which the Si-rich silicon oxide $\left(\mathrm{SiO}_{x}, x<2\right)$ sublayers are sandwiched between $\mathrm{SiO}_{2}$ sublayers allowing to control the size of the Si nanocluster formed during the growth and/or after specific annealing treatment [88-90]. Thin films, composite structures, multilayers, powders, aggregates, and other systems have been
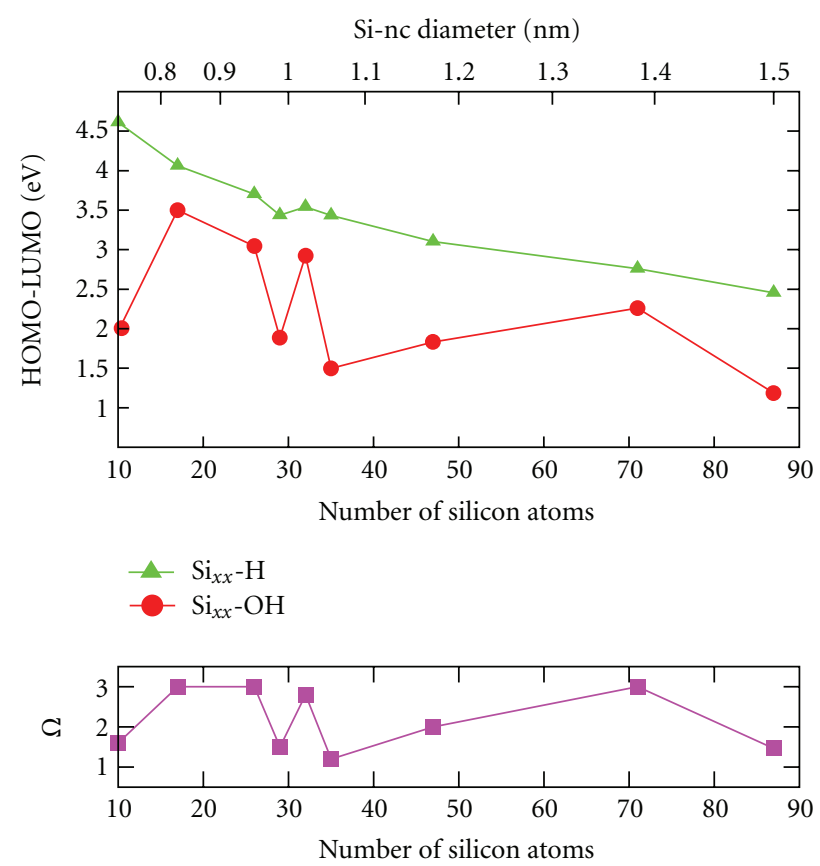

FIGURE 3: HOMO-LUMO gaps for the hydrogenated (triangles) and hydroxided (circle) nanocrystals together with the oxidation degree $\Omega$ (squares) as function of the Si-nc diameter [60].

extensively analyzed and deeply studied by means of several characterization techniques to understand and to enhance the emission properties of the Si nanostructures.

3.1.2. Optical Wedges and Free-Standing Films. Sometimes, it is useful to study $\mathrm{SiO}_{x}$ samples with the Si concentration varying along the film surface [91, 92]. One such sample contains areas with different composition but otherwise treated under the same conditions. These samples are called optical wedges because the different areas possess different optical thickness. Interference patterns are seen by the naked eye from these samples showing gradient of optical thickness. The results obtained with these samples will be described below.

Free-standing films were from films on silicon substrates annealed at $1100^{\circ} \mathrm{C}$ in nitrogen atmosphere in a furnace. A part of the substrate was carefully removed by an etching procedure [93]. The free-standing films were typically 0.5 to $2 \mu \mathrm{m}$ thick and up to several millimeters wide, and they were supported by silicon frames. The thinnest free-standing films were directly suitable for transmission electron microscopy (TEM) measurements, which is a very interesting research opportunity as also seen below.

3.2. Characterization Methods and Basic Observations. A key point for a full understanding of the intriguing optical properties of Si-nc is the availability of a clear picture of their structural properties. This task is made more complicated by the complex evolution induced by the annealing process (in a furnace, in most of cases), which is usually required to increase the light emission efficiency $[79,86]$. In the large amount of the literature data dealing with characterization 
of Si-nc, analytical techniques such as transmission electron microscopy (TEM) [79, 88, 93-97], X-ray diffraction (XRD) $[88,95,96]$, XPS [86], and Raman and PL spectroscopy $[86,95]$ are widely employed.

3.2.1. TEM Characterization. Fairly reliable estimations of the Si-nc size have been obtained by TEM $[79,97]$ although this technique is not able to give a complete quantitative picture of the system. Indeed, the most used analysis modes (dark field and high resolution) are blind to the presence of amorphous Si clusters. Furthermore, high resolution measurements, being very time consuming, are intrinsically inadequate to provide statistically significant measurements of the Si-nc distribution. On the other hand, the dark field TEM (DFTEM) technique, although being statistically more reliable, is able to detect only a small fraction of the crystalline population [98]. These limitations introduce some errors in the estimation of the Si-nc mean size and, above all, prevent the access to fundamental information such as the Si-nc volume density. As a consequence, a meaningful correlation between the structural and optical properties of Si-nc (and, above all, information about the role played by amorphous clusters) can be hardly provided by the conventional TEM modes.

The energy filtered TEM (EFTEM) technique allows a more complete characterization of the structural properties of Si-nc, owing to its capability to provide a chemical mapping with the very high spatial resolution typical of TEM [94]. As an example, Figures 4(a) and 4(b) show the EFTEM images of a $\mathrm{SiO}_{x}$ film deposited by PECVD and then annealed at 900 and $1000^{\circ} \mathrm{C}$ for $1 \mathrm{~h}$ in $\mathrm{N}_{2}$ environment, respectively. The brighter regions of the images are associated with Si clusters; their presence demonstrates that clustering effects are already visible at $900^{\circ} \mathrm{C}$. The $\mathrm{Si}-\mathrm{SiO}_{2}$ phase separation becomes much more evident at higher annealing temperatures, and well-defined Si clusters embedded in an oxide matrix are visible in the sample annealed at $1000^{\circ} \mathrm{C}$. The absence of any cluster in the corresponding dark field images demonstrates that, at these temperatures, although an extensive phase separation is already occurring, no crystallization process takes place.

As another example, the comparison between an EFTEM image and a conventional TEM image obtained by DFTEM is shown in Figures 4(c) and 4(d). The sample is a $\mathrm{SiO}_{x}$ film deposited by PECVD and then annealed at $1250^{\circ} \mathrm{C}$ for $1 \mathrm{~h}$ in $\mathrm{N}_{2}$ environment. The comparison between the two images clearly demonstrates the advantages of EFTEM as compared with the dark field technique. Indeed, while in the conventional TEM image only a small portion of the crystalline population is shown, the EFTEM image is able to detect both crystalline and amorphous clusters.

3.2.2. Raman and PL Measurements. Raman spectroscopy is a powerful method to study the structure of solid-state materials. This technique can distinguish between crystalline and amorphous phased and shed light on chemical composition and bonding. It is important that Raman measurements can be easily performed in vacuum and at low and elevated temperatures and do not require special sample preparation.
In some cases, in situ Raman measurements can be performed during sample preparation. In studies of Si-based photonic materials, it is important that Raman measurements can be run simultaneously with PL measurements.

Examples of Raman and PL spectra of Si-based materials are shown in Figure 5 [9]. The $\mathrm{SiO}_{x}(x=1.7)$ film was prepared on a silica substrate by MBD. Before annealing, the material contained amorphous $\mathrm{Si}$ inclusions as shown by the broad Raman band around $470 \mathrm{~cm}^{-1}$. This broad band decreases in intensity, and a narrow band at $\sim 517 \mathrm{~cm}^{-1}$ appears after annealing the sample above $1000^{\circ} \mathrm{C}$, which shows silicon crystallization. The formed Si-nc have typical diameters of 3-4 $\mathrm{nm}$ as estimated by TEM [93]. Simultaneously with $\mathrm{Si}$ crystallization, the PL intensity strongly increases and somewhat redshifts. This correlation between Raman and PL spectra seemingly suggests that the PL directly originates from the Si-nc observed by Raman spectroscopy. However, the case is much more complex as mentioned in the computational section and further discussed below.

3.2.3. XPS Measurements. The XPS measurements provide detailed information about chemical composition and bonding. XPS is surface sensitive meaning that the analysis layer is very thin. In order to investigate the bulk, the sample can be sputtered layer by layer with $\mathrm{Ar}^{+}$ions. The Si $2 p$ spectrum can be fitted by several peaks corresponding to the Si oxidation states. For simplicity, the fitting is often limited by three peaks for the silicon binding energy in crystalline silicon (elemental silicon), silicon suboxides $(\mathrm{SiO})$, and silicon dioxide $\left(\mathrm{SiO}_{2}\right)$ [99]. In this case, the silicon suboxide ( $\mathrm{SiO}$ ) component represents a mixture of various suboxides. For a $\mathrm{SiO}_{x}$ sample $(x=1.7)$ annealed at $1100^{\circ} \mathrm{C}$, the XPS method gives only a few percent of elemental silicon, whereas the largest part of the Si excess appears to be in suboxides [99]. This result shows the lack of $\mathrm{Si}-\mathrm{SiO}_{2}$ phase separation in this material, and this conclusion is valid for furnace annealing at temperatures at least up to $1200^{\circ} \mathrm{C}$.

3.2.4. Optical Measurements. Optical properties of Si-rich silicon oxide films can be measured by using spectral filtering of PL $[92,100,101]$. When the PL excited in a relatively thick absorbing layer is detected from the sample edge along the surface (waveguiding geometry), the PL spectrum consists of a number of sharp and polarized peaks (see Figure 6). This spectral filtering was explained by delocalization of guided light near the cutoff frequency $[92,100,101]$ and by leaky (substrate) modes [102]. The peak positions show the waveguide cutoff frequencies that depend on the optical properties of the system. The comparison of the transmission and cutoff spectra allows one to find the refractive index and layer thickness $[92,100,101]$. The layer thickness is used to calculate the absorption coefficient from the transmission measurements. Figure 7 shows the correlation between the absorption coefficient at $488 \mathrm{~nm}$ and refractive index for $\mathrm{SiO}_{x}$ ( $x$ from 1.35 to 1.75 ) films annealed at $1100^{\circ} \mathrm{C}$ [92]. These results were confirmed by m-line measurements, which yielded very similar optical properties of these samples, including optical birefringence of Si-nc [103]. 


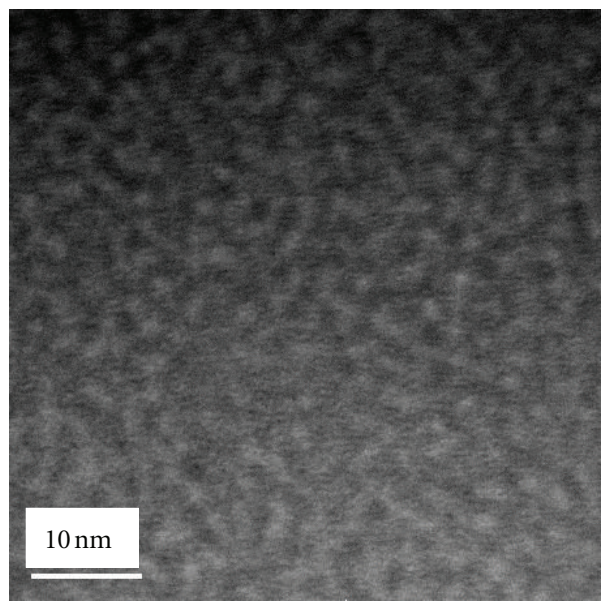

(a)

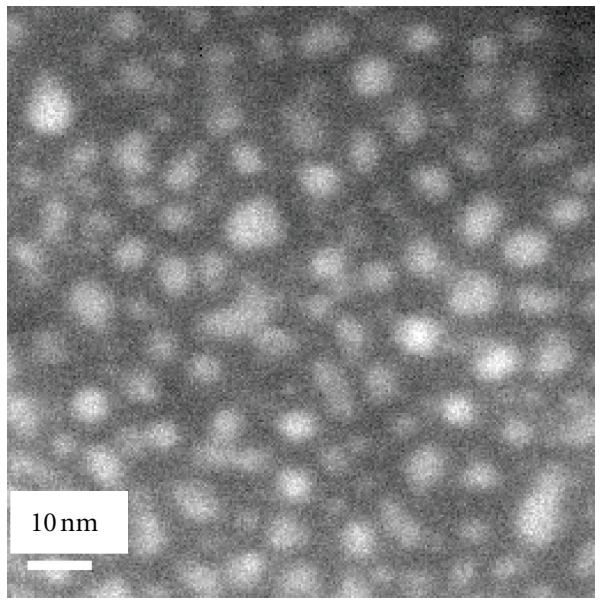

(c)

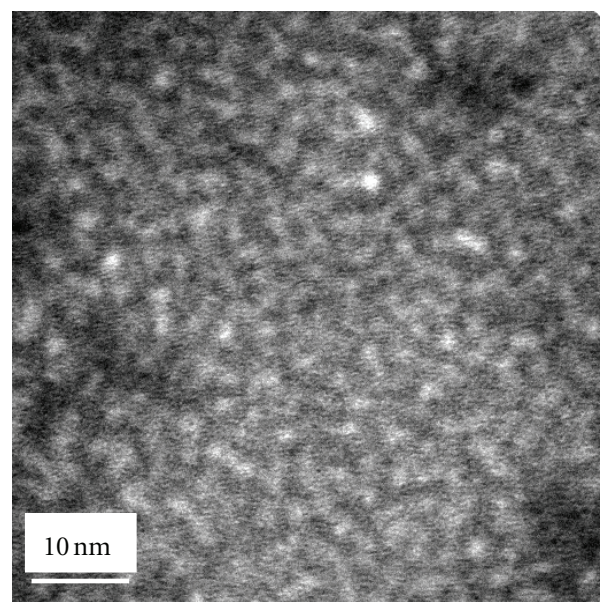

(b)

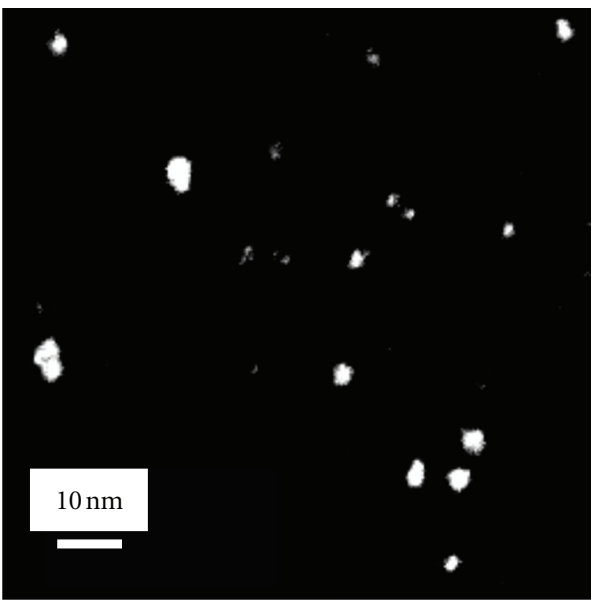

(d)

Figure 4: EFTEM plan view images obtained by selecting the Si plasmon loss electrons for $\mathrm{SiO}_{x}$ films annealed at (a) $900^{\circ} \mathrm{C}$, (b) $1000^{\circ} \mathrm{C}$, and (c) $1250^{\circ} \mathrm{C}$. (d) DFTEM plan view image obtained from a $\mathrm{SiO}_{x}$ film annealed at $1250^{\circ} \mathrm{C}$.

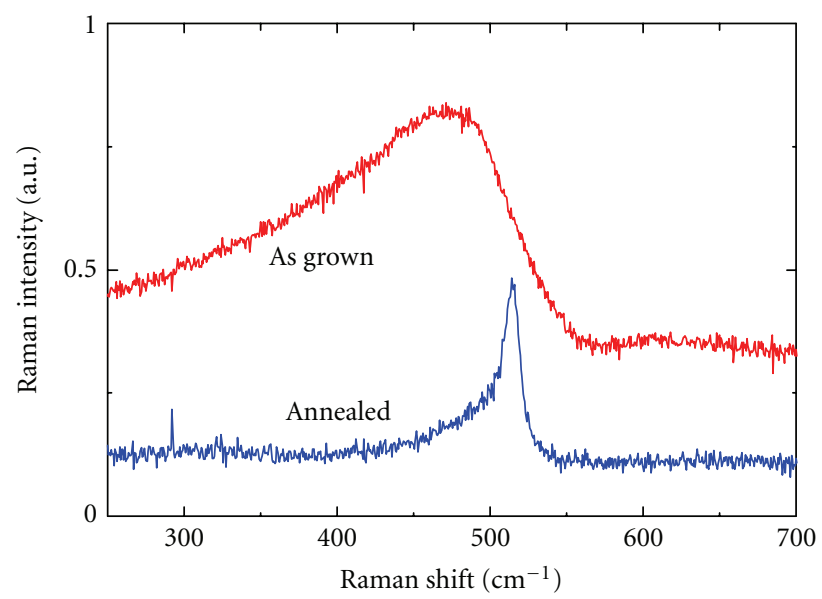

(a)

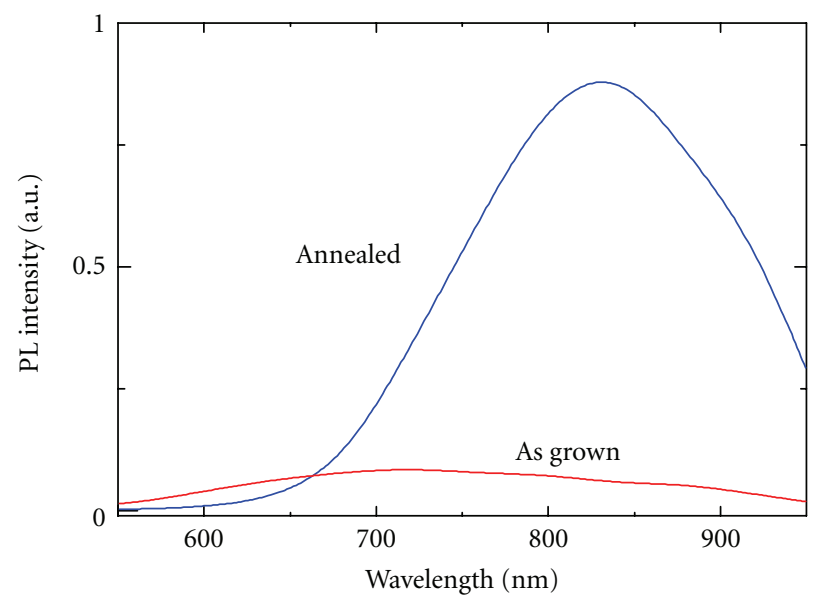

(b)

Figure 5: Raman (a) and PL (b) spectra of as grown and annealed at $1100^{\circ} \mathrm{C} \mathrm{SiO}_{x}(x=1.7)$ films [9]. 


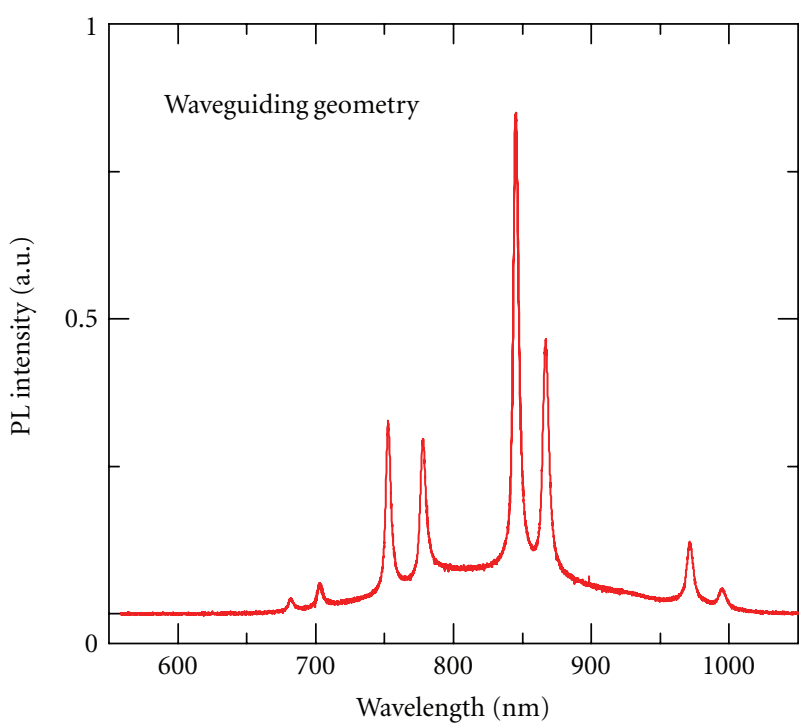

Figure 6: PL from a $\mathrm{SiO}_{x}$ film measured in the waveguiding geometry, that is, along the surface from the film edge [100].

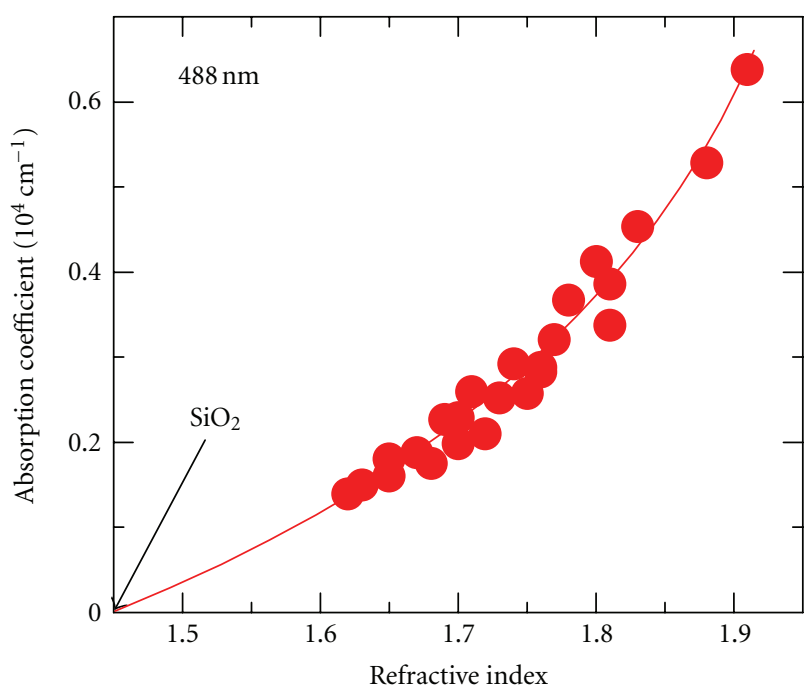

Figure 7: Correlation between the absorption coefficient at $488 \mathrm{~nm}$ and refractive index of $\mathrm{SiO}_{x}$ films with different $\mathrm{Si}$ excess [92].

3.2.5. Atom Probe Tomography (APT). This is a powerful analytical tool to investigate materials with an atomic resolution in three dimensions (3D) which allows precise analysis of semiconductors and insulating materials like $\mathrm{Si}$ nanostructures. The basic principle of this method is the field evaporation of surface atoms on a sharp tip. The APT is a projection microscope with magnification larger than a few millions. A complete review and historical improvements of atom probe and sample preparation can be found elsewhere [104-107]. Nowadays, this technique is increasingly used for semiconductor and insulating materials [108-110].

The research group of the Rouen University (Physic of Materials Group) has performed APT analysis on photonic and photovoltaic Si-based materials. An example of the 3D reconstruction of $\mathrm{Si}$ nanoparticles in silica and the $\mathrm{Si}$ and $\mathrm{O}$ concentration profiles are presented in Figure 8 [108]. The sample was a stacking sequence of $\mathrm{SiO}_{x}(4 \mathrm{~nm}) / \mathrm{SiO}_{2}$ $(4 \mathrm{~nm})$ layers $(x \sim 1)$ deposited on a Si substrate by reactive magnetron sputtering. After deposition, the sample was annealed at $900^{\circ} \mathrm{C}$ for $1 \mathrm{~h}$. The presence of pure $\mathrm{Si}$ nanoparticles and the multilayered structure are clearly demonstrated and resolved.

The 3D atomic resolution of APT allows one to investigate individual clusters (Figure 9). Talbot and coworkers have deduced from APT experiments major results on the structural properties of Si-nc $[108,110]$. It has been shown that Si-nc are pure and with an abrupt interface with the surrounding matrix. Moreover, for this sample annealed at $900^{\circ} \mathrm{C}$ for $1 \mathrm{~h}$, the composition of the matrix (i.e., without $\mathrm{Si}$ nc contribution) is not stoichiometric silica, but it is close to $\mathrm{SiO}_{x}$ with $x=1.4$ evidencing an incomplete phase separation process in the sample. These data shed light on the phase separation process and the growth mechanism of Si-nc which are required to control the size and then to tune the Si band gap for new solar devices.

\section{Main Properties}

4.1. Phase Separation in $\mathrm{SiO}_{x}$ Films. The microstructure of the $\mathrm{SiO}_{x}$ films can be described as a simple $\mathrm{Si} / \mathrm{SiO}_{2}$ mixture (random mixture model, RMM [111]) or, alternatively, as a mixture of all the Si oxidation states, under the form of $\mathrm{Si}-\mathrm{Si}_{x} \mathrm{O}_{4-x}$ tetrahedra $(0 \leq x \leq 4)$ where $\mathrm{O}$ atoms are replaced by $\mathrm{Si}$ atoms in order to respect the film stoichiometry (random bonding model, RBM [112]). RMM is based on the assumption that the growth of $\mathrm{a} \mathrm{SiO}_{x}$ film is mainly ruled by chemical forces; only the more stable phases, $\mathrm{Si}$ and $\mathrm{SiO}_{2}$, are formed, while the existence of the intermediate $\mathrm{Si}$ oxidation states is neglected. On the contrary, RBM is a purely statistical model, in which the $\mathrm{Si}-\mathrm{Si}_{x} \mathrm{O}_{4-x}$ tetrahedra distribution depends only on the frequency of arrival of $\mathrm{O}$ and $\mathrm{Si}$ atoms on the substrate surface during deposition and, therefore, on their relative concentrations in the gaseous phase. Both "pure" models are never experimentally verified, and usually the $\mathrm{SiO}_{x}$ structure is intermediate between these two extreme situations. Deposition temperature plays a fundamental role if the film structure is more similar to RMM or to RBM. In films deposited at high temperature, a heat-induced ordering, from a statistics-driven structure to a chemistry-driven one, occurs, and the film grows is in good agreement with the RMM. This effect takes place even if the energy needed to the system to reorganize itself according to chemical forces is supplied after the deposition. The thermal evolution of the film structure involves the formation of a biphasic mixture where $\mathrm{Si}$ coexists with a $\mathrm{SiO}_{x}$ phase whose composition is closer to the stoichiometry than that present in the as-deposited film. We can schematically depict the heat-induced disproportionation reaction undergone by the partially oxidized $\mathrm{Si}-\mathrm{Si}_{x} \mathrm{O}_{4-x}$ tetrahedra as follows:

$\mathrm{Si}-\mathrm{Si}_{x} \mathrm{O}_{4-x} \longrightarrow \frac{x}{4} \mathrm{Si}_{-} \mathrm{Si}_{4}+\left(1-\frac{x}{4}\right) \mathrm{Si}-\mathrm{O}_{4} \quad$ (with $\left.x=1,2,3\right)$, 


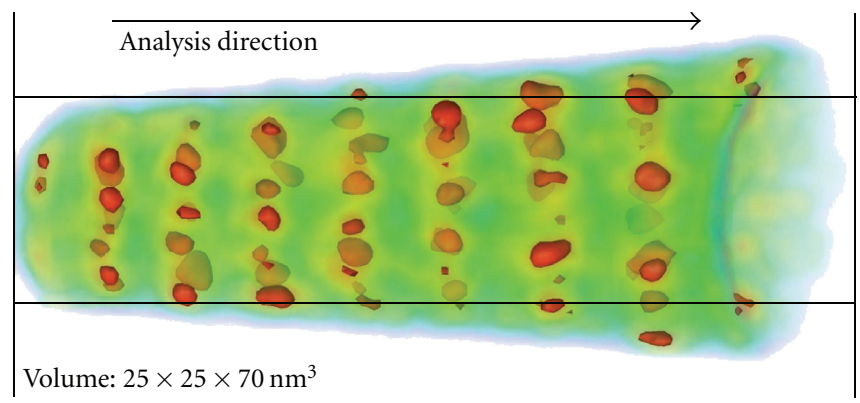

(a)

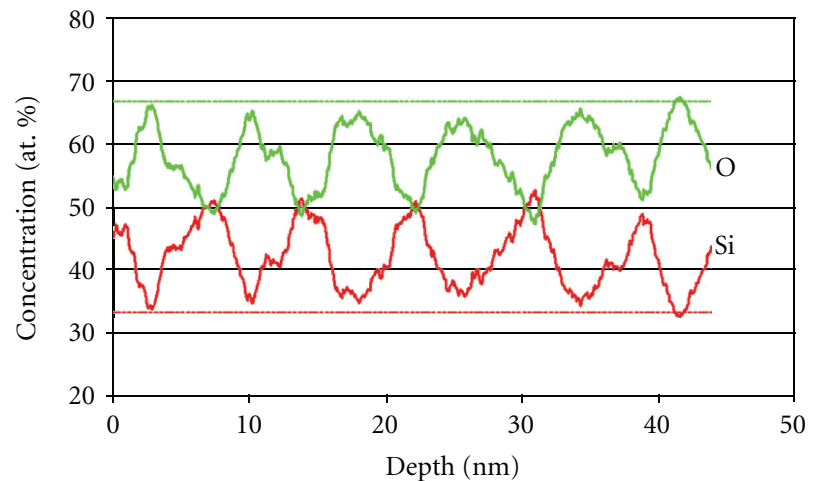

(b)

Figure 8: (a) 3D reconstruction of APT analysis on $\mathrm{Si}-\mathrm{nc}-\mathrm{SiO}_{2} / \mathrm{SiO}_{2}$ multilayers. (b) Composition profile along the growth direction.

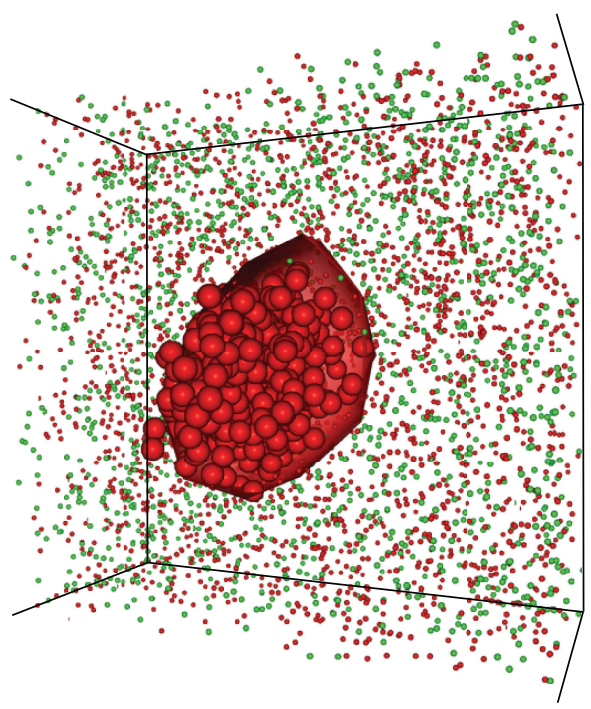

FIGURE 9: 3D atomic mapping of an individual Si-nc in silica matrix. Each dot corresponds to one atom ( $\mathrm{Si}$ in red, $\mathrm{O}$ in green). The size of $\mathrm{Si}$ atoms belonging to $\mathrm{Si}-\mathrm{nc}$ is artificially increased. The shown volume is $8 \times 8 \times 8 \mathrm{~nm}^{3}$.

where the $\mathrm{Si}_{-} \mathrm{Si}_{4}$ tetrahedra constitute the building blocks for the formation of Si-nc.

The thermal evolution of $\mathrm{SiO}_{x}$ films from the amorphous mixture of $\mathrm{Si}$ and $\mathrm{O}$ atoms in deposited films to the formation of Si-nc embedded in $\mathrm{SiO}_{2}$ can be successfully studied by combining dark field and energy filtered TEM analyses. The size of the Si nanoclusters detected in plan view EFTEM images of films annealed at temperatures ranging from 1000 to $1250^{\circ} \mathrm{C}$ is reported in Figure 10 (a) as a function of the annealing temperature; the mean radii increase with the annealing temperature from $1.0\left(\right.$ at $1000^{\circ} \mathrm{C}$ ) to $2.6 \mathrm{~nm}$ (at $1250^{\circ} \mathrm{C}$ ). The DFTEM technique, which is sensitive to the presence of crystalline planes, has been used to map the system for the presence of Si-nc that have been detected only in samples annealed at temperatures above $1100^{\circ} \mathrm{C}$. To estimate the crystalline fraction, images obtained by DFTEM (showing only Si-nc) and EFTEM (showing all
Si clusters) have been compared. The data have been reported in Figure 10(b) and demonstrate the continuous increase of the crystalline fraction with the annealing temperature. Assuming that all clusters are crystalline at $1250^{\circ} \mathrm{C}$ [98], the estimation of the $\mathrm{Si}-\mathrm{nc}$ fraction at lower temperatures leads to values of about $30 \%$ at $1100^{\circ} \mathrm{C}$ (the temperature at which the crystallization process starts) and $60 \%$ at $1150^{\circ} \mathrm{C}$, clearly demonstrating that in this range, the temperature plays a role not only by promoting the Si nanocluster growth but also by inducing the amorphous to crystal transition.

Important information that can be derived from plan view EFTEM images is the volume density of Si nanoclusters at the different temperatures. Under suitable experimental conditions [94, 98], EFTEM can detect all the clusters present in a given volume. Typical density values are from $10^{17}$ to $10^{18} \mathrm{~cm}^{-3}$; by coupling these data with those on the crystalline fraction reported in Figure 10(b), we can calculate the concentration of $\mathrm{Si}-\mathrm{nc}$ and of amorphous clusters as a function of the annealing temperature. The data are reported in Figure 10(c) and demonstrate that the temperature progressively induces the complete transformation of the amorphous clusters $\left(9 \times 10^{17} \mathrm{~cm}^{-3}\right.$ at $\left.1000^{\circ} \mathrm{C}\right)$ into Si-nc $\left(7 \times 10^{17} \mathrm{~cm}^{-3}\right.$ at $\left.1250^{\circ} \mathrm{C}\right)$, with the loss of about $20 \%$ of the clusters present at $1000^{\circ} \mathrm{C}$, probably due to the occurrence of Ostwald ripening phenomena.

From the data on the cluster density and mean radius, the number of clustered Si atoms per unit volume at the different temperatures can be calculated. The amount of Si atoms forming detectable clusters remarkably increases with the annealing temperature, but a substantial fraction of excess $\mathrm{Si}$ atoms is not detectable by EFTEM even after annealing at $1250^{\circ} \mathrm{C}$. Indeed, the value of $3 \times 10^{21} \mathrm{~cm}^{-3}$ measured for this temperature has to be compared with an expected value for the excess Si content, deduced from the Rutherford backscattering measurements, of about $1 \times 10^{22} \mathrm{~cm}^{-3}$. This conclusion is in qualitative agreement with the XPS data indicated a substantial volume of $S i$ suboxides after annealing at $1200^{\circ} \mathrm{C}$ [99].

The behaviour of the annealed $\mathrm{SiO}_{x}$ films described above is qualitatively similar for all $\mathrm{SiO}_{x}$ films prepared by PECVD although the clustering and crystallization 


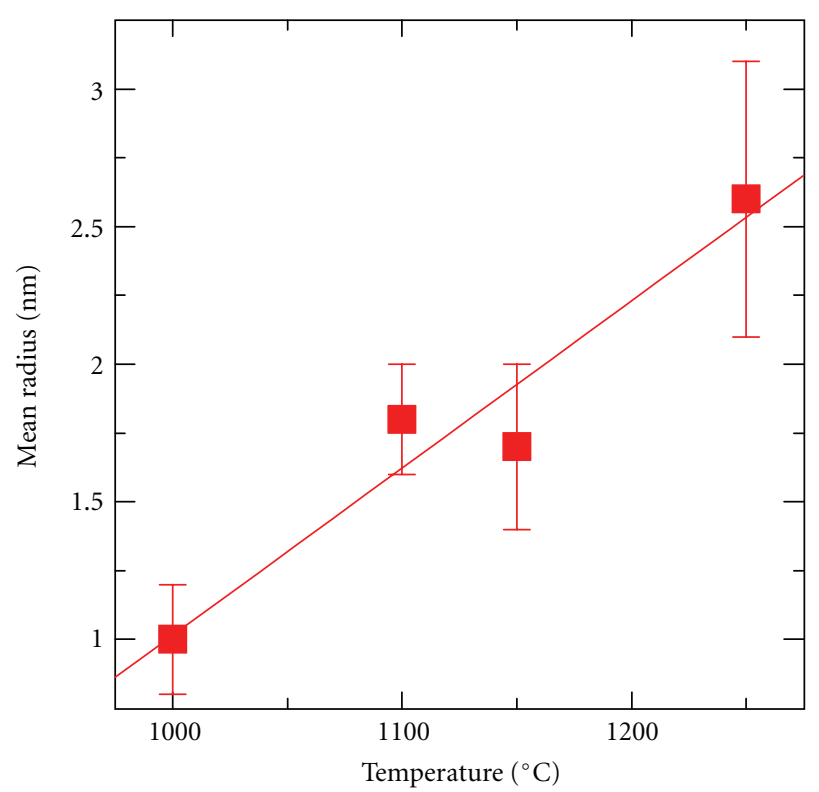

(a)

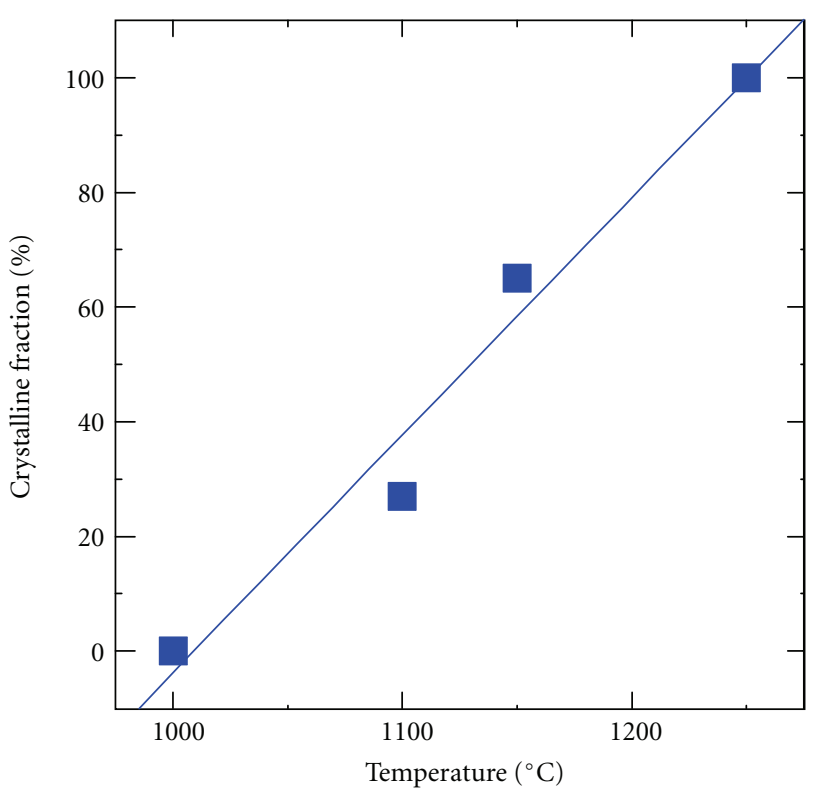

(b)

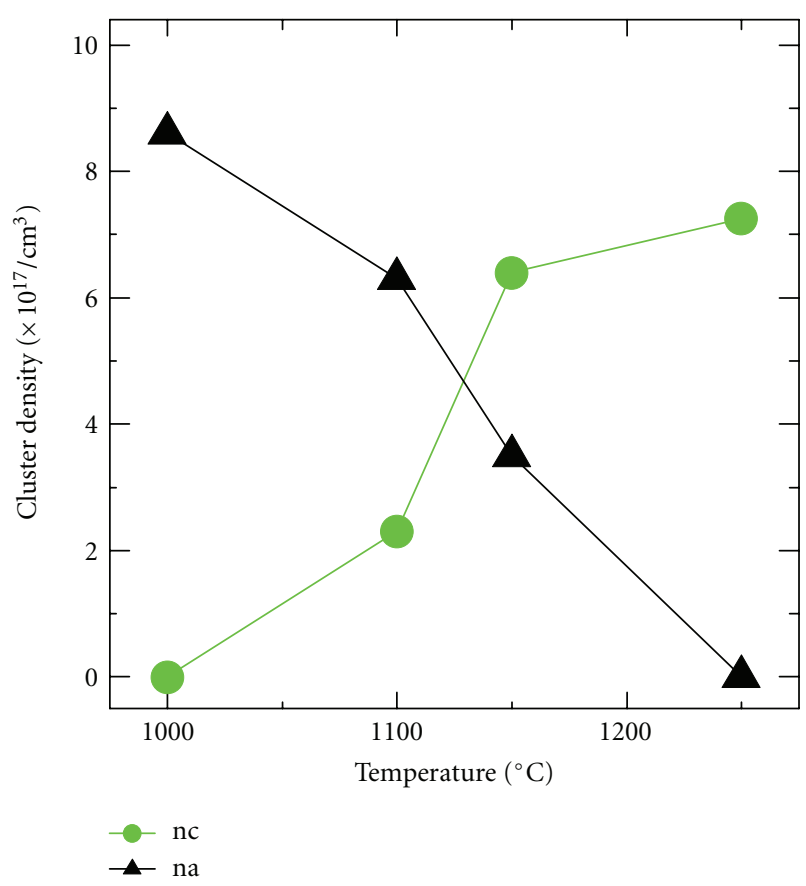

(c)

FIGURE 10: (a) Si nanocluster mean radius obtained from EFTEM images, (b) crystalline fraction obtained from the comparison of EFTEM and DFTEM images, and (c) density of amorphous (na) and crystalline (nc) nanoclusters, as a function of the annealing temperature. The lines are drawn to guide the eye.

temperatures may vary depending on the composition [79]. On the other hand, very deep structural differences can be found in $\mathrm{SiO}_{x}$ films having the same composition but prepared by different deposition techniques. The most striking example is the almost complete clustering of the $\mathrm{Si}$ excess found in $\mathrm{SiO}_{x}$ films prepared by magnetron sputtering [113].
4.2. Correlation between Optical and Structural Properties. The as-grown $\mathrm{SiO}_{x}$ films with a substantial $\mathrm{Si}$ excess prepared by MBD exhibit broad Raman bands around $470 \mathrm{~cm}^{-1}$, which is an indication of amorphous $\mathrm{Si}$ inclusions (see Figure 5(a)). A similar spectrum is obtained for as-grown $\mathrm{Si} / \mathrm{SiO}_{2}$ superlattices (SLs) with relatively thick Si layers. For low $\mathrm{Si}$ excess in $\mathrm{SiO}_{x}$ films $(x \sim 1.9)$ and very thin silicon 
layers $(\sim 1 \mathrm{~nm})$ in $\mathrm{Si} / \mathrm{SiO}_{2}$ SLs, the $470 \mathrm{~cm}^{-1}$ Raman band of amorphous silicon is not seen $[86,114,115]$. These results were interpreted in terms of efficient Si oxidation for small $\mathrm{Si}$ excess, and this is consistent with the optical properties obtained for the same samples. The refractive index and extinction coefficient of ultrathin Si layers were found to differ from the corresponding values of bulk silicon. These differences show that silicon inclusions interact with the oxide host, resulting in the formation of suboxide interfaces with different optical properties. It was extracted that $\mathrm{Si}$ and $\mathrm{SiO}_{2}$ amorphous layers in a $\mathrm{Si} / \mathrm{SiO}_{2} \mathrm{SL}$ are separated by $\sim 0.8 \mathrm{~nm}$ thick suboxide interfaces with $n=2$ and very low absorption [115].

Thermal annealing above $1000^{\circ} \mathrm{C}$ leads to progressive crystallization of Si inclusions as indicated by the narrowing and upshifting of Raman bands (see Figure 5). Based on the phonon confinement model, the $\sim 517 \mathrm{~cm}^{-1}$ Raman bands correspond to Si-nc with diameters of 3-4 nm [116], which is in excellent agreement with the TEM results [93]. A broader shoulder at lower energy may be an indication of smaller crystallites (diameters $<3 \mathrm{~nm}$ ). The lack of Raman fingerprints of ultrasmall silicon grains $(<2 \mathrm{~nm})$ does not necessarily mean their absence because they may be invisible by Raman spectroscopy. It should be stressed that the integrated Raman intensity of Si-nc after thermal annealing is much smaller than the value expected from the Si excess.

The structure of the annealed samples can be influenced by the substrate material. In fact, thin $\mathrm{Si} / \mathrm{SiO}_{2} \mathrm{SLs}$ on silicon wafers have upshifted and narrowed Raman bands compared to similar SLs on silica substrates [117]. This difference was connected with annealing-induced stress due to the different thermal expansion coefficients of the film and substrate, and simple estimates agree well with the experimental data. The broader Raman bands obtained for the films on silica substrates were connected with a wider distribution of stresses. The PL spectra also depend on the substrate material.

The XPS measurements were performed for different $\mathrm{SiO}_{x}$ films prepared by MBD [99]. When $x=\mathrm{O} / \mathrm{Si}$ ratio increases from 1.35 to 1.75 , the $\mathrm{SiO}_{2}$ amount increases and the elemental Si amount decreases, whereas the amount of suboxides is rather stable. Analysis shows a very good agreement between XPS and Raman results, that is, the Raman signal of $\mathrm{Si}-\mathrm{nc}$ is proportional to the amount of elemental silicon provided by XPS. A similarly good agreement is observed between the absorption coefficient measured at $488 \mathrm{~nm}$ and the amount of elemental silicon.

Based on the XPS data, a large part of the Si excess after annealing at $1100^{\circ} \mathrm{C}$ is in various suboxides [99]. However, this seemingly definite interpretation should be commented. The concept of a crystal lattice is not straightforward in the case of ultra-small Si grains in a silica matrix. The proportion of bulk-like coordinated $\mathrm{Si}$ atoms substantially decreases for smaller grains, and the binding energy may change. This raises a question about the applicability of the XPS method for the analysis of ultra-small Si grains $(<1 \mathrm{~nm})$. It is possible that these features are hardly distinguishable from suboxides, similarly to the case of Raman spectroscopy and absorption measurements. At this stage, we can only claim that the elemental silicon obtained by XPS produces the optical absorption and the Raman signal. Some part of the $\mathrm{Si}$ excess is not visible in Raman and absorption measurements, and it is consumed in suboxides and/or in very small and possibly amorphous Si grains. As a reminder, some part of the $\mathrm{Si}$ excess is also invisible in the TEM images as pointed out above, which can be due to the presence of suboxides and/or very small sizes of Si clusters [98].

Optical properties of these $\mathrm{SiO}_{x}$ samples were analysed using the Bruggeman effective medium approximation and the structural components obtained from the XPS measurements [99]. The refractive index was found to be well predicted by using the properties of bulk materials; however, some artificial assumptions should be made to obtain the experimental extinction coefficients. In fact, high transparency of the suboxide component should be assumed, in contrast to bulk suboxides absorbing light. The elemental silicon should be taken with the extinction coefficient, which is between the values for crystalline and amorphous silicon, which shows incomplete crystallization of Si particles. These complications may also be an indication of the presence of very small Si grains that are optically transparent but have a substantially high refractive index.

4.3. Laser-Induced Thermal Effects. Intense laser light can increase temperature in absorbing materials and lead to structural changes. This fact should be kept in mind while studying Si-based materials with powerful lasers. On the other hand, laser irradiation can be used to produce desired results in sample processing. The heating effect is especially strong for pulsed lasers, but it can be also sufficient for continuous wave (CW) lasers especially with microscopes although this effect is very often neglected. The laser-induced temperature becomes much stronger for free-standing films that allow high steady-state temperatures due to the lack of thermal contact with the substrate [118].

Various laser heat effects on free-standing $\mathrm{SiO}_{x}$ films have been studied [32, 119-121]. A short exposure of a free-standing film to focused laser light produces structural changes; in particular, the Raman intensity of Si-nc strongly increases (see Figure 11). The observed structural changes were connected with high temperatures produced by laser light [119]. The laser-induced temperatures were estimated by comparing the anti-Stokes and Stokes Raman bands. It was found that the temperature is a nearly linear function of the $488 \mathrm{~nm}$ laser power, and high temperatures $(\sim 1300 \mathrm{~K}$ and higher) are easily produced with a laser power of $\sim 100 \mathrm{~mW}$. Above $\sim 1300 \mathrm{~K}$, the Raman measurements are practically impossible due to strong thermal radiation.

Si-nc produced by laser annealing are under compressive stress as evidenced by the upshift of the Raman bands with respect to crystalline silicon (high-temperature annealing, HTA, in Figure 11) [32, 121]. The first experiments were done on $\mathrm{Si} / \mathrm{SiO}_{2} \mathrm{SLs}$, but all conclusions are unchanged for $\mathrm{SiO}_{x}$ films. An exposure to lower laser intensity (lowtemperature annealing, LTA) shifts the Raman band gradually to lower frequencies. In terms of stress, the difference of the HTA and LTA band positions corresponds to about $3 \mathrm{GPa}[122]$. The up-shift of Raman bands (compressive 


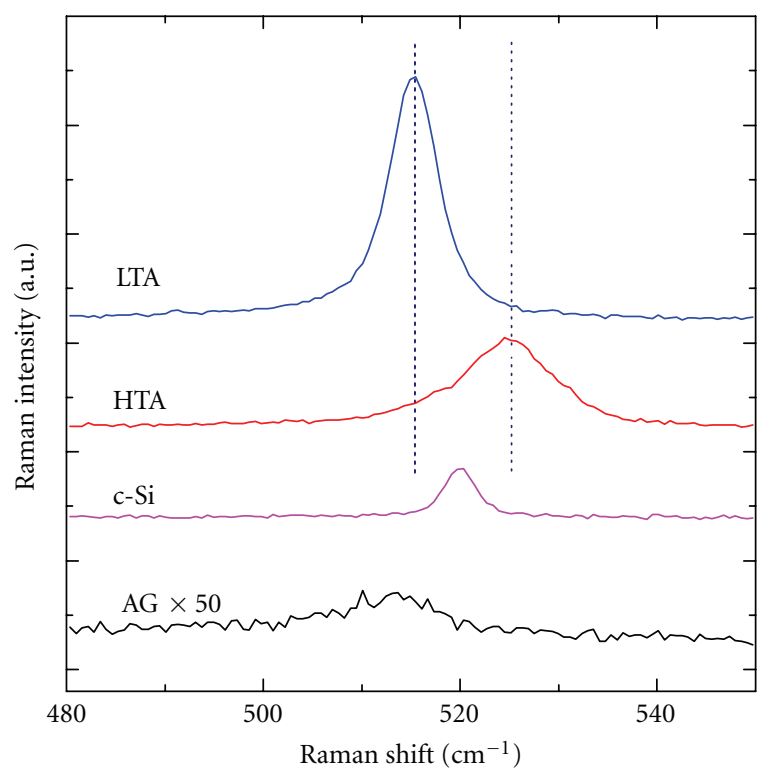

Figure 11: (From bottom to top) Raman spectra of an as-grown $\mathrm{Si} / \mathrm{SiO}_{2}$ superlattice free-standing film (AG), crystalline $\mathrm{Si}$ (c-Si), free-standing film after high-temperature laser annealing (HTA), and subsequent low-temperature laser annealing (LTA).

stress) occurs at temperatures close to silicon melting point $(1685 \mathrm{~K})$. In the proposed model, laser annealing above the silicon melting point (HTA) melts silicon inclusions and releases stresses in the vicinity. Crystallization of a Si particle from the liquid phase increases its volume by $\sim 10 \%$ [123], which evidently leads, in a solid silica matrix, to a compressive stress. The numerical estimates for spherical Si particles in a silica matrix produced excellent agreement with experiment [32]. As shown later [124], the assumptions used in those simple estimates were very adequate to the experimental reality, but in the first work published in 2006 these were lucky guesses. The relaxation of Si-nc stress was investigated at different temperatures. The slope of the Arrhenius plot yields an activation energy of $(1.6 \pm 0.2) \mathrm{eV}[32,121]$; however, the exact mechanism of this process is unknown.

A more precise look at the laser-annealed area was taken by a combination of various microscopic methods such as Raman and FTIR spectroscopy, TEM, EELS, and XPS [124]. In general, three regions were observed on a free-standing $\mathrm{SiO}_{x}$ film after laser exposure: central region, ring around the central region, and pristine film outside the laser-annealed area. The Raman map of Si-nc overlaps with the ring region seen in an optical microscope. The optical transmission is maximum in the central region and strongly decreases in the ring. Most importantly, the central region (up to $30 \mu \mathrm{m}$ wide) is practically free of Si excess. The material here is essentially $\mathrm{SiO}_{2}$ as shown by the XPS and EELS measurements, and the results from other methods are consistent with this conclusion. The ring around the central region contains large spherical Si-nc (diameters up to $\sim 100 \mathrm{~nm}$ ) embedded in amorphous $\mathrm{SiO}_{2}$ without the presence of suboxides. The strong Raman signal is obtained in this region, and the large Si-nc are under compressive stress after HTA. The XPS and
TEM data show an increase of the Si excess in the ring region as compared with the pristine film. Thus, these results demonstrate macroscopic (long range) $\mathrm{Si}-\mathrm{SiO}_{2}$ phase separation, that is, the $\mathrm{Si}$ excess is moved from the central to ring region over a distance of tens of micrometers. The process was explained by extensive diffusion in temperature gradient leading to the Si concentration gradient (thermodiffusion) [125]. It should be stressed that laser annealing also leads to complete $\mathrm{Si}-\mathrm{SiO}_{2}$ phase separation in the ring region because no presence of suboxides is observed, which is different from furnace annealing.

4.4. EL Properties of Si-nc. One of the most relevant properties of Si-nc is the possibility to excite them electrically. Indeed, this property opens the way to the possibility of fabricating light-emitting devices with electrical excitation. $\mathrm{SiO}_{x}$ films are conductive due to the $\mathrm{Si}$ excess, which allows electrical current to flow through them when properly biased. The main mechanisms of conduction are tunneling (either direct or Fowler-Nordheim [16]) or Poole-Frenkel [126]. Excitation of Si-nc mainly occurs through the impact excitation by hot electrons [16].

Light-emitting devices based on Si-nc embedded in $\mathrm{SiO}_{2}$ with a simple MOS structure have been successfully fabricated; the top electrode is an $n$-type polysilicon film, the active region is a $\mathrm{SiO}_{x}$ film, and a highly doped p-type $\mathrm{Si}$ substrate acts as the bottom electrode. Aluminum contacts to the electrodes have a circular shape and define a metalfree central area for the exit of the light. The emission of these devices was obtained by emission microscopy (EMMI) measurements (see Figure 12(a)). The EMMI image is in false colors and reveals a bright emission, very stable and homogeneous over the whole active area. The emission typically starts at a voltage of about $15 \mathrm{~V}$, but a proper choice of the layer containing Si-nc remarkably decreases the operating voltage down to $5 \mathrm{~V}$ [17]. In this point of view, the most relevant parameters are the Si-nc size and density, their amorphous or crystalline nature, and the concentration of residual excess $\mathrm{Si}$ dissolved in the matrix, which can be controlled by playing on the film composition and/or the annealing temperature. Also the film thickness has an important role $[16,17]$.

Spectral analysis of the light emitted from the devices has been performed. Figure 12(b) shows a typical room temperature EL spectrum; it consists of two peaks: a weak one at $660 \mathrm{~nm}$, which can be attributed to the presence of radiative defects in the oxide matrix and an intense one at $890 \mathrm{~nm}$. The main EL peak is very similar both in position and shape to the PL peak measured in the same sample and shown for comparison in the same figure. Therefore, the mechanisms of EL and PL seem to be the same [16]. An intense and stable EL emission at $1.54 \mu \mathrm{m}$ has been obtained from devices having a structure very similar to that one described above, but in which the active layer contains Erdoped Si nanoclusters [27].

A new device structure for an optimized EL from Si-nc has been proposed [127]. To improve the carrier injection efficiency, a field-effect EL mechanism has been employed. In this excitation process, electrons and holes are sequentially 


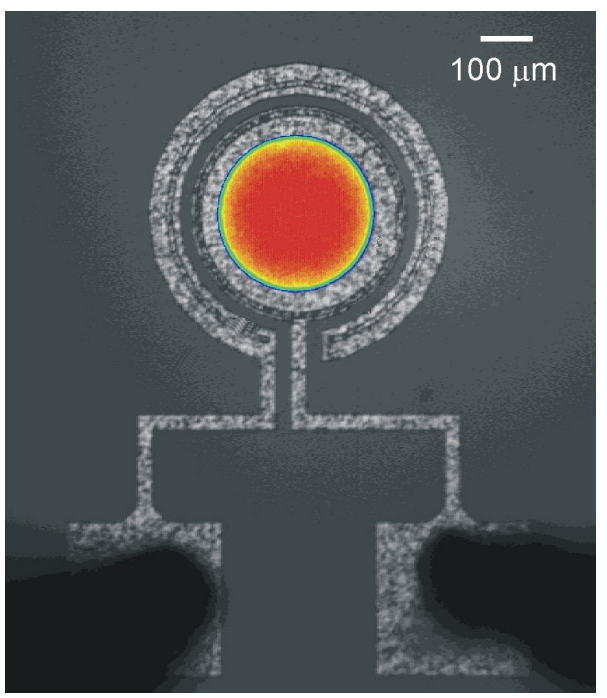

(a)

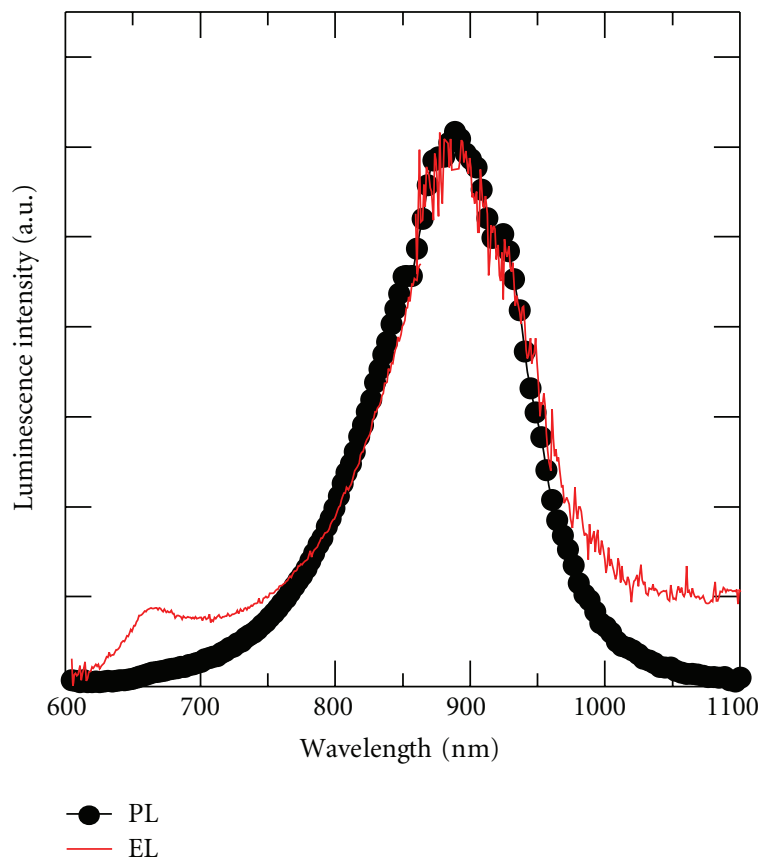

(b)

Figure 12: (a) EMMI image of a device based on Si-nc. The colors indicate different intensities of the emitted light, red being the highest. (b) Comparison between the PL and the EL spectra of the device.

injected in array of Si-nc embedded in the gate oxide of a transistor. The formed excitons recombine radiatively giving origin to the EL signal. This approach is expected to reduce nonradiative processes limiting the EL efficiency, such as Auger recombination, as well as to improve the device reliability since it involves a less extended oxide wear-out with respect to the impact excitation by hot carriers. An enhanced light extraction from a light-emitting device based on Si nanostructures has been demonstrated by coupling a two-dimensional photonic crystal with the device [128].
4.5. Light Emission Mechanism. This subject remains controversial despite a large number of theoretical and experimental works trying to evaluate it. Excess of $\mathrm{Si}$ in silicon oxide and annealing treatment are essential factors to obtain the $1.5 \mathrm{eV}$ PL. It was found long ago that in general this PL does not follow quantum confinement criteria for electronhole pair recombination in Si-nc, and surface-state model was suggested. In this model, light comes from the nearsurface region of small crystallites [129]. By studying porous silicon, Wolkin et al. suggested that the light emission could originate from $\mathrm{Si}=\mathrm{O}$ bonds [10], and this model received support from theoretical analysis [130]. The model of $\mathrm{Si}=\mathrm{O}$ bonds was also used in connection to Si-nc in silica matrix [86]. It was suggested later that the light-emitting centers can be stabilized not only at Si-nc surface but also in a disordered network [91]. In accord, Godefroo et al. have confirmed that small defects are the dominant source of the red PL from Si-nc in silica [131]; however, the structure of these defects was not identified. Recent simulations have suggested that the bridge $\mathrm{Si}-\mathrm{O}-\mathrm{Si}$ bonds are more suitable to explain light emission from these systems compared to the $\mathrm{Si}=\mathrm{O}$ double bonds [54].

As described in the theoretical section, very small oxidized Si grains can produce strong light emission in this spectral region [62]. It seems that this proposition is consistent with many experimental results. The XPS data show practically no elemental silicon for $\mathrm{SiO}_{x}$ samples with $x>1.75$, that is, no large Si-nc (several $\mathrm{nm}$ in diameter); however, relatively strong PL is measured for these samples [99]. These highly luminescent samples are very transparent, and practically no Raman bands of Si-nc are detected. Moreover, anticorrelation between the Raman and PL intensity was found [91]. This fact shows that the observed red PL is not from Si-nc with sizes $3-4 \mathrm{~nm}$ as often claimed in the literature. Furthermore, it appears that the PL quantum yield strongly decreases when the amount of elemental Si increases for $x$ from $\sim 1.75$ to 1.35 (see Figure 13) [75]. These results show that Si-nc measured by XPS as elemental silicon, visible by Raman spectroscopy, and producing light absorption are not the direct light-emitting phase of the annealed $\mathrm{SiO}_{x}$ material. It follows that the lightemitting centers are oxygen-related defects and/or ultrasmall Si grains. They probably locate in the suboxides where the excitation migrates from the absorbing Si phase, and the exciton transfer can be suppressed by elemental silicon. It is also possible that the light-emitting centers can be directly excited by light.

\section{Some Perspective Applications}

5.1. Light-Emitting Devices Based on Si Nanoclusters. The efficiency of the EL emission from devices based on Si-nc is still lower than that required for practical applications, presumably due to competing nonradiative processes. The EL quenching processes competing with the radiative emission in devices based on Er-doped Si nanoclusters have been investigated in detail, demonstrating that Auger deexcitation with trapped electrons, although absent during $\mathrm{PL}$ experiments, represents the main non-radiative process 


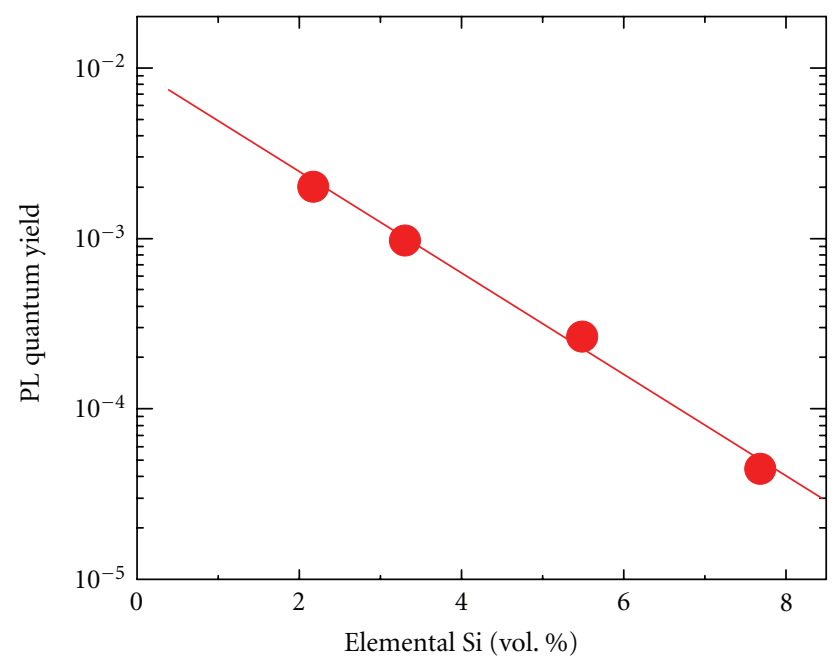

FIGURE 13: PL quantum yield (in arbitrary units) as a function of the amount of elemental $\mathrm{Si}$ in $\mathrm{SiO}_{x}$ films [75].

under electrical pumping [29]. When dealing with Si-nc or Er-doped Si nanoclusters, it is necessary to consider the key role played by the matrix in which they are embedded. Indeed, when Si-nc are electrically excited, the characteristics of the matrix influence not only their emission properties, as widely established for the optical excitation but also their conduction properties and, in turn, their excitation efficiency. Most of the literature contributions reporting EL from Si-nc refer to systems generated by thermal annealing of $\mathrm{SiO}_{x}$ films grown by PECVD. This technique has a peculiarity; there is a strong limitation on the fraction of excess Si which can be actually clustered, even for very high annealing temperatures. In particular, only about $30 \%$ of the excess $\mathrm{Si}$ is able to form clusters, while the most remain "dissolved" in the matrix, which has to be considered $\mathrm{SiO}_{x}$ rather than stoichiometric $\mathrm{SiO}_{2}$. The recent demonstration that, on the contrary, an almost complete Si clusterization can be accomplished in $\mathrm{SiO}_{x}$ films synthesized by sputter deposition [113] makes available for device fabrication also $\mathrm{Si}$-nc embedded in a nearly stoichiometric $\mathrm{SiO}_{2}$ matrix. In particular, the use of the reactive sputtering technique markedly improves the quality of the oxide matrix, leading to efficient and stable LEDs. Furthermore, the presence of a stoichiometric oxide matrix avoids the trapping of the carriers in the active layer and the consequent occurring of Auger processes between the trapped carriers and the excited Er ions, which represents the main limitation to the efficiency of LEDs based on Si-nc-containing layers synthesized by PECVD. The evidence that Auger deexcitation can be efficiently suppressed in Er-doped Si nanocluster layers synthesized by reactive sputter deposition may represent a further step towards the implementation of this material in LEDs having real perspectives for practical applications in Si nanophotonics [30].

Another strong limitation to the performances of light sources based on Si nanostructures is related to the efficiency of extraction of the emitted photons. Indeed, in a typical $\mathrm{Si}$ LED, due to the high Si refractive index $(n=3.5)$, most of the emitted light remains trapped inside the device by total internal reflection. The realization of a two-dimensional photonic crystal $(\mathrm{PhC})$ on the device surface can overcome this limitation by enabling the extraction of a large amount of light, which would be otherwise wasted, thus, enhancing the overall efficiency [128]. A two-dimensional $\mathrm{PhC}$ has been simply integrated with the devices described above by patterning the polysilicon upper electrode with conventional UV lithography. The triangular $\mathrm{PhC}$ is composed of holes with a diameter of $400 \mathrm{~nm}$ and has a lattice constant of $900 \mathrm{~nm}$. The depth of the holes is $200 \mathrm{~nm}$, leaving an uninterrupted $100 \mathrm{~nm}$ thick polysilicon layer for the electrical contact with the active layer. The lattice constant of the PhC has been tuned in order to introduce a fourthorder diffraction escape path at $860 \mathrm{~nm}$. In fact, in resonance with the designed diffraction spot of the two dimensional photonic pattern, a sharp emission peak at around $860 \mathrm{~nm}$ was observed [128]. The evaluation of the ratio between the EL intensity of the device with the $\mathrm{PhC}$ and a similar device without $\mathrm{PhC}$ demonstrates that the photonic structure enhances the intensity of the emission by a factor of four at $860 \mathrm{~nm}$. These results can open the way to a whole new generation of Si-based devices in which photonic and electronic functions are integrated together.

5.2. Photovoltaics. Since the discovery, in the 90s, of the quantum confinement effect in Si nanostructures, these latter have attracted great interest in the photonic application during the last 20 years and more recently in the photovoltaic applications. Such interest is due to the optical properties but also to the promising electrical ones coupled to the overcome of the Si bulk restricted conversion efficiency. One of the advantages to work with $\mathrm{Si}$ nanoclusters is the ability to monitor the band gap of the material by varying the size of the Si-nc embedded in a dielectric matrix. This band gap tuning coupled to the increase of the absorption efficiency of the light in a compatible integrated Si technology process offers interesting perspectives for the third generation of solar cells whose aim is to decrease the cost of a cell below \$1/W [132]. Different approaches have been developed during these last years to increase the conversion efficiency of the silicon-based solar cells. They concern the following: (i) the increase of the light trapping using silicon nanowires [133], silicon nanoholes [134], or surface plasmon resonance $[135,136]$, (ii) the use of a downshifter layer based on Si nanostructures [137], (iii) the multiexciton generation process in Si nanostructures [138], and (iv) the tandem solar cell [139]. This latter approach is based on the stack of two different band gap layers. In an all-Si tandem solar cell, the proposed structure consists of a Si-based nanostructured thin film absorbing around $1.7 \mathrm{eV}$ deposited on the classical photovoltaic Si cell. One of the most promising structures is the $\mathrm{SiO}_{x} / \mathrm{SiO}_{2}$ multilayer because it allows accurate control of the Si-nc size as well as the Si-nc density; these both parameters being critical for the future performance of the solar cell [97]. Moreover, such a structure permits to overcome partially the limitation of the electrical properties by decreasing the dielectric layer thickness in the range of the tunnel effect [140]. Nevertheless, up to now, high-resistivity 
device and low-output voltage have only been achieved with this approach. This is why new structures have been recently developed and studied where $\mathrm{SiO}_{2}$ layers are replaced by either $\mathrm{Si}_{3} \mathrm{~N}_{4}$ or $\mathrm{SiN}_{x}$ layers $[139,141]$. These structures demonstrate good absorption $\left(\sim 10^{5} \mathrm{~cm}^{-1}\right.$ at $\left.3.5 \mathrm{eV}\right)$ as well as a strong decrease of the resistivity of the $\mathrm{SiO}_{x} / \mathrm{SiN}_{y}$ multilayers with respect to the $\mathrm{SiO}_{x} / \mathrm{SiO}_{2}$ system [142]. Lately, a conversion efficiency of about $10 \%$ has been achieved in an $n$-type Si quantum dots/ $p$-type crystalline Si solar cell at $298 \mathrm{~K}$ [143]. The studies on nanostructured Si based thin films for solar cell application are actually an open field and give rise to wide range of promising developments for which important disruptive results will probably be achieved in the next five years.

5.3. Optical Memory. Optical memory seems to be one of the most complicated tasks for Si nanophotonics. The observed laser-annealing effect on free-standing films containing Sinc makes a case of permanent optical memory readable by means of Raman spectroscopy [119]. The obtained change is detectable also by a camera photographs and transmission measurements [124]. This change can be made in a very small spots of submicron size, which estimates the achievable data density [144]. The structural change done by laser annealing is persistent at very high temperatures, at least at $1200^{\circ} \mathrm{C}$, showing the superior thermal stability. It is important that such optical memory is realised within conventional Si technology. However, these optical data cannot be erased.

The laser-controlled stress of Si-nc provides an approach to rewritable optical memory [32]. The demonstrated effect allows writing and erasing information and its reading by optical means, which is principally provided by means of micro-Raman mapping. This approach also offers a very long retention time. Raman microscopy was used to estimate the spatial limitations [144]. An extended area with compressive stress was first prepared and then locally relaxed by sharply focused laser light. As a result, the spot with relaxed stress had dimensions as small as $\sim 2 \mu \mathrm{m}$. The obtained optical change cannot be seen by camera photographs and transmission measurements, but it is detectable by Raman spectroscopy. This approach is potentially useful for nonvolatile data storage elements produced within silicon technology. Nevertheless, extensive technological work is needed to move the present experimental observations to practical applications. Different materials and irradiation by pulsed lasers can probably provide better performance.

\section{Concluding Remarks}

We have described a part of activities in the area of silicon photonics, in most cases working essentially on nanoscale. We have presented the analytical methods and some of the key results in the area. A number of optical functions are possible based on Si-nc, such as light emission, amplification, modulation, waveguiding, and optical memory. In many cases, the current research concentrates on the visible spectral region, which is perspective for photonic application.
Many of the results and properties can be simulated and understood based on theoretical studies. However, some of the key properties like the light-emitting mechanism are subjects of intense debates despite a remarkable progress in the recent years. For example, high efficiency of luminescence has been recently found for small oxidized Si clusters, which shows an alternative for the quantum confinement mechanism of the light emission. Evidently, additional theoretical studies and models are needed to fully understand the responsible mechanisms at the molecular level.

Even more complex is to move the known experimental observations towards practical applications. The demonstrated devices and approaches are often too complex (impractical) and/or possess too low efficiency. However, the challenge to realize the optical functions on a chip and effectively combine optical and electrical function is very strong. This explains why we expect more activity in the field of Si nanophotonics in the future.

\section{Acknowledgments}

The authors thank all colleagues who have contributed to the described results. L. Khriachtchev thanks the Finnish Centre of Excellence in Computational Molecular Science for partial support of this work.

\section{References}

[1] S. Ossicini, L. Pavesi, and F. Priolo, Light Emitting Silicon for Microphotonics. Springer Tracts on Modern Physics, vol. 194, Springer, Berlin, Germany, 2003.

[2] L. Pavesi and D. J. Lockwood, Eds., Silicon Photonics, Springer, 2004.

[3] L. Khriachtchev, Ed., Silicon Nanophotonics: Basic Principles, Present Status and Perspectives, Pan Stanford, Singapore, 2008.

[4] N. Koshida, Ed., Device Applications of Silicon Nanocrystals and Nanostructures, Springer, 2008.

[5] L. Pavesi and R. Turan, Eds., Silicon Nanocrystals: Fundamentals, Synthesis and Applications, Wiley, 2010.

[6] L. T. Canham, "Silicon quantum wire array fabrication by electrochemical and chemical dissolution of wafers," Applied Physics Letters, vol. 57, no. 10, pp. 1046-1048, 1990.

[7] A. G. Cullis, L. T. Canham, and P. D. J. Calcott, "The structural and luminescence properties of porous silicon," Journal of Applied Physics, vol. 82, no. 3, pp. 909-965, 1997.

[8] L. Pavesi, L. Dal Negro, C. Mazzoleni, G. Franzò, and F. Priolo, "Optical gain in silicon nanocrystals," Nature, vol. 408, no. 6811, pp. 440-444, 2000.

[9] L. Khriachtchev, M. Räsänen, S. Novikov, and J. Sinkkonen, "Optical gain in $\mathrm{Si} / \mathrm{SiO}_{2}$ lattice: experimental evidence with nanosecond pulses," Applied Physics Letters, vol. 79, no. 9, pp. 1249-1251, 2001.

[10] M. V. Wolkin, J. Jorne, P. M. Fauchet, G. Allan, and C. Delerue, "Electronic states and luminescence in porous silicon quantum dots: the role of oxygen," Physical Review Letters, vol. 82, no. 1, pp. 197-200, 1999.

[11] D. J. DiMaria, J. R. Kirtley, E. J. Pakulis et al., "Electroluminescence studies in silicon dioxide films containing tiny silicon islands," Journal of Applied Physics, vol. 56, no. 2, pp. 401-416, 1984. 
[12] G. G. Qin, A. P. Li, B. R. Zhang, and B. C. Li, "Visible electroluminescence from semitransparent $\mathrm{Au}$ film/extra thin Si-rich silicon oxide film/p-Si structure," Journal of Applied Physics, vol. 78, no. 3, pp. 2006-2009, 1995.

[13] S. Fujita and N. Sugiyama, "Visible light-emitting devices with Schottky contacts on an ultrathin amorphous silicon layer containing silicon nanocrystals," Applied Physics Letters, vol. 74, no. 2, pp. 308-310, 1999.

[14] P. Photopoulos and A. G. Nassiopoulou, "Room- and lowtemperature voltage tunable electroluminescence from a single layer of silicon quantum dots in between two thin $\mathrm{SiO}_{2}$ layers," Applied Physics Letters, vol. 77, no. 12, pp. 1816-1818, 2000.

[15] N. Lalic and J. Linnros, "Light emitting diode structure based on Si nanocrystals formed by implantation into thermal oxide," Journal of Luminescence, vol. 80, no. 1-4, pp. 263-267, 1998.

[16] G. Franzò, A. Irrera, E. C. Moreira et al., "Electroluminescence of silicon nanocrystals in MOS structures," Applied Physics A, vol. 74, no. 1, pp. 1-5, 2002.

[17] A. Irrera, D. Pacifici, M. Miritello et al., "Excitation and deexcitation properties of silicon quantum dots under electrical pumping," Applied Physics Letters, vol. 81, no. 10, pp. 18661868, 2002.

[18] G. Franzò, F. Priolo, S. Coffa, A. Polman, and A. Carnera, "Room-temperature electroluminescence from Er-doped crystalline Si," Applied Physics Letters, vol. 64, no. 17, pp. 2235-2237, 1994.

[19] G. Franzò, S. Coffa, F. Priolo, and C. Spinella, "Mechanism and performance of forward and reverse bias electroluminescence at $1.54 \mu \mathrm{m}$ from Er-doped Si diodes," Journal of Applied Physics, vol. 81, no. 6, pp. 2784-2793, 1997.

[20] A. J. Kenyon, P. F. Trwoga, M. Federighi, and C. W. Pitt, "Optical properties of PECVD erbium-doped siliconrich silica: evidence for energy transfer between silicon microclusters and erbium ions," Journal of Physics Condensed Matter, vol. 6, no. 21, pp. L319-L324, 1994.

[21] M. Fujii, M. Yoshida, Y. Kanzawa, S. Hayashi, and K. Yamamoto, "1.54 $\mu \mathrm{m}$ photoluminescence of $\mathrm{Er}^{3+}$ doped into $\mathrm{SiO}_{2}$ films containing $\mathrm{Si}$ nanocrystals: evidence for energy transfer from Si nanocrystals to $\mathrm{Er}^{3+}$," Applied Physics Letters, vol. 71, no. 9, pp. 1198-1200, 1997.

[22] J. H. Shin, M. J. Kim, S. Y. Seo, and C. Lee, "Composition dependence of room temperature $1.54 \mu \mathrm{m} \mathrm{Er}^{3+}$ luminescence from erbium-doped silicon:oxygen thin films deposited by electron cyclotron resonance plasma enhanced chemical vapor deposition," Applied Physics Letters, vol. 72, no. 9, pp. 1092-1094, 1998.

[23] G. Franzò, V. Vinciguerra, and F. Priolo, "Excitation mechanism of rare-earth ions in silicon nanocrystals," Applied Physics A, vol. 69, no. 1, pp. 3-12, 1999.

[24] P. G. Kik, M. L. Brongersma, and A. Polman, "Strong excitonerbium coupling in $\mathrm{Si}$ nanocrystal-doped $\mathrm{SiO}_{2}$," Applied Physics Letters, vol. 76, no. 17, pp. 2325-2327, 2000.

[25] F. Priolo, G. Franzò, D. Pacifici, V. Vinciguerra, F. Iacona, and A. Irrera, "Role of the energy transfer in the optical properties of undoped and Er-doped interacting Si nanocrystals," Journal of Applied Physics, vol. 89, no. 1, pp. 264-272, 2001.

[26] F. Gourbilleau, M. Levalois, C. Dufour, J. Vicens, and R. Rizk, "Optimized conditions for an enhanced coupling rate between Er ions and Si nanoclusters for an improved 1.54$\mu \mathrm{m}$ emission," Journal of Applied Physics, vol. 95, no. 7, pp. 3717-3722, 2004.
[27] F. Iacona, D. Pacifici, A. Irrera et al., "Electroluminescence at $1.54 \mu \mathrm{m}$ in Er-doped Si nanocluster-based devices," Applied Physics Letters, vol. 81, no. 17, pp. 3242-3244, 2002.

[28] A. Nazarov, J. M. Sun, W. Skorupa et al., "Light emission and charge trapping in Er-doped silicon dioxide films containing silicon nanocrystals," Applied Physics Letters, vol. 86, no. 15, Article ID 151914, 2005.

[29] F. Priolo, C. D. Presti, G. Franzò et al., "Carrier-induced quenching processes on the erbium luminescence in silicon nanocluster devices," Physical Review B, vol. 73, no. 11, Article ID 113302, 2006.

[30] A. Irrera, F. Iacona, G. Franzò et al., "Influence of the matrix properties on the performances of Er-doped Si nanoclusters light emitting devices," Journal of Applied Physics, vol. 107, no. 5, Article ID 054302, 2010.

[31] G. Conibeer, M. Green, E. C. Cho et al., "Silicon quantum dot nanostructures for tandem photovoltaic cells," Thin Solid Films, vol. 516, no. 20, pp. 6748-6756, 2008.

[32] L. Khriachtchev, M. Räsänen, and S. Novikov, "Lasercontrolled stress of Si nanocrystals in a free-standing $\mathrm{Si} / \mathrm{SiO}_{2}$ superlattice," Applied Physics Letters, vol. 88, no. 1, Article ID 013102, 2006.

[33] C. Delerue and M. Lannoo, Nanostructuresl Theory and Modelling, Springer, Berlin, Germany, 2004.

[34] A. Di Carlo, "Microscopic theory of nanostructured semiconductor devices: beyond the envelope-function approximation," Semiconductor Science and Technology, vol. 18, no. 1, pp. R1-R31, 2003.

[35] P. Harrison, Quantum Wells, Wires and Dots, Wiley, New York, NY, USA, 2nd edition, 2005.

[36] D. M. Wood and A. Zunger, "Successes and failures of the $\mathrm{k} \cdot \mathrm{p}$ method: a direct assessment for GaAs/AlAs quantum structures," Physical Review B, vol. 53, no. 12, pp. 7949-7963, 1996.

[37] L. W. Wang, A. Franceschetti, and A. Zunger, "Million-atom pseudopotential calculation of $\Gamma-\mathrm{X}$ mixing in GaAs/AlAs superlattices and quantum dots," Physical Review Letters, vol. 78, no. 14, pp. 2819-2822, 1997.

[38] C. Bulutay, "Electronic structure and optical properties of silicon nanocrystals along their aggregation stages," Physica E, vol. 38, no. 1-2, pp. 112-117, 2007.

[39] C. Bulutay, "Interband, intraband, and excited-state direct photon absorption of silicon and germanium nanocrystals embedded in a wide band-gap lattice," Physical Review B, vol. 76, no. 20, Article ID 205321, 2007.

[40] H. Yildirim and C. Bulutay, "Enhancement of optical switching parameter and third-order optical nonlinearities in embedded Si nanocrystals: a theoretical assessment," Optics Communications, vol. 281, pp. 4118-4120, 2008.

[41] C. Sevik and C. Bulutay, "Auger recombination and carrier multiplication in embedded silicon and germanium nanocrystals," Physical Review B, vol. 77, no. 12, Article ID 125414, 2008.

[42] E. Degoli and S. Ossicini, "Engineering quantum confined silicon nanostructures: ab-initio study of the structural, electronic and optical properties," Advances in Quantum Chemistry, vol. 58, pp. 203-279, 2009.

[43] E. Degoli, R. Guerra, F. Iori et al., "Ab-initio calculations of luminescence and optical gain properties in silicon nanostructures," Comptes Rendus Physique, vol. 10, no. 6, pp. 575-586, 2009. 
[44] P. Hohenberg and W. Kohn, "Inhomogeneous electron gas," Physical Review, vol. 136, no. 3, pp. B864-B871, 1964.

[45] W. Kohn and L. J. Sham, "Self-consistent equations including exchange and correlation effects," Physical Review, vol. 140, no. 4, pp. A1133-A1138, 1965.

[46] L. Hedin, "New method for calculating the one-particle Green's function with application to the electron-gas problem," Physical Review, vol. 139, no. 3, pp. A796-A823, 1965.

[47] G. Onida, L. Reining, and A. Rubio, "Electronic excitations: density-functional versus many-body Green's-function approaches," Reviews of Modern Physics, vol. 74, no. 2, pp. 601-659, 2002.

[48] E. Runge and E. K. U. Gross, "Density-functional theory for time-dependent systems," Physical Review Letters, vol. 52, no. 12, pp. 997-1000, 1984.

[49] L. E. Ramos, J. Paier, G. Kresse, and F. Bechstedt, "Optical spectra of Si nanocrystallites: Bethe-Salpeter approach versus time-dependent density-functional theory," Physical Review $B$, vol. 78, no. 19, Article ID 195423, 2008.

[50] A. Puzder, A. J. Williamson, J. C. Grossman, and G. Galli, "Computational studies of the optical emission of silicon nanocrystals," Journal of the American Chemical Society, vol. 125, no. 9, pp. 2786-2791, 2003.

[51] M. Luppi and S. Ossicini, "Multiple $\mathrm{Si}=\mathrm{O}$ bonds at the silicon cluster surface," Journal of Applied Physics, vol. 94, no. 3, pp. 2130-2132, 2003.

[52] M. Gatti and G. Onida, "Effects of local fields in timedependent density functional theory shown in oxidized silicon clusters," Physical Review B, vol. 72, no. 4, Article ID 045442, 2005.

[53] I. Vasiliev, J. R. Chelikowsky, and R. M. Martin, "Surface oxidation effects on the optical properties of silicon nanocrystals," Physical Review B, vol. 65, no. 12, Article ID 121302, 2002.

[54] E. Luppi, F. Iori, R. Magri et al., "Excitons in silicon nanocrystallites: the nature of luminescence," Physical Review B, vol. 75, no. 3, Article ID 033303, 2007.

[55] Z. Ma, X. Liao, G. Kong, and J. Chu, "Absorption spectra of nanocrystalline silicon embedded in $\mathrm{SiO}_{2}$ matrix," Applied Physics Letters, vol. 75, no. 13, pp. 1857-1859, 1999.

[56] N. Daldosso, M. Luppi, S. Ossicini et al., "Role of the interface region on the optoelectronic properties of silicon nanocrystals embedded in $\mathrm{SiO}_{2}$," Physical Review B, vol. 68, no. 8, Article ID 085327, 2003.

[57] M. Luppi and S. Ossicini, "Ab initio study on oxidized silicon clusters and silicon nanocrystals embedded in $\mathrm{SiO}_{2}$ : beyond the quantum confinement effect," Physical Review B, vol. 71, no. 3, Article ID 035340, 2005.

[58] R. Guerra, I. Marri, R. Magri et al., "Silicon nanocrystallites in a $\mathrm{SiO}_{2}$ matrix: role of disorder and size," Physical Review $B$, vol. 79, no. 15, Article ID 155320, 2009.

[59] R. Guerra, I. Marri, R. Magri et al., "Optical properties of silicon nanocrystallites in $\mathrm{SiO}_{2}$ matrix: crystalline versus amorphous case," Superlattices and Microstructures, vol. 46, no. 1-2, pp. 246-252, 2009.

[60] R. Guerra, E. Degoli, and S. Ossicini, "Size, oxidation, and strain in small $\mathrm{Si} / \mathrm{SiO}_{2}$ nanocrystals," Physical Review B, vol. 80, no. 15, Article ID 155332, 2009.

[61] R. Guerra, E. Degoli, M. Marsili, O. Pulci, and S. Ossicini, "Local-fields and disorder effects in free-standing and embedded Si nanocrystallites," Physica Status Solidi B, vol. 247, no. 8, pp. 2113-2117, 2010.
[62] R. Guerra and S. Ossicini, "High luminescence in small $\mathrm{Si} / \mathrm{SiO}_{2}$ nanocrystals: a theoretical study," Physical Review B, vol. 81, no. 24, Article ID 245307, 2010.

[63] T. Watanabe, K. Tatsumura, and I. Ohdomari, " $\mathrm{SiO}_{2} / \mathrm{Si}$ interface structure and its formation studied by large-scale molecular dynamics simulation," Applied Surface Science, vol. 237, no. 1-4, pp. 125-133, 2004.

[64] P. Kroll and H. J. Schulte, "Shell-like structure of valence band orbitals of silicon nanocrystals in silica glass," Physica Status Solidi B, vol. 243, no. 6, pp. R47-R49, 2006.

[65] D. E. Yilmaz, C. Bulutay, and T. Çağin, "Pathways of bond topology transitions at the interface of silicon nanocrystals and amorphous silica matrix," Physical Review B, vol. 77, no. 15, Article ID 155306, 2008.

[66] G. Hadjisavvas and P. C. Kelires, "Structure and energetics of Si nanocrystals embedded in $\mathrm{a}_{-} \mathrm{SiO}_{2}$," Physical Review Letters, vol. 93, Article ID 226104, 2007.

[67] G. Hadjisavvas and P. C. Kelires, "Theory of interface structure, energetics, and electronic properties of embedded $\mathrm{Si} / \mathrm{a}-\mathrm{SiO}_{2}$ nanocrystals," Physica E, vol. 38, no. 1-2, pp. 99105, 2007.

[68] M. Ippolito, S. Meloni, and L. Colombo, "Interface structure and defects of silicon nanocrystals embedded into a- $\mathrm{SiO}_{2}$," Applied Physics Letters, vol. 93, no. 15, Article ID 153109, 2008.

[69] F. Djurabekova and K. Nordlund, "Atomistic simulation of the interface structure of Si nanocrystals embedded in amorphous silica," Physical Review B, vol. 77, no. 11, Article ID 115325, 2008.

[70] B. Gallas, I. Stenger, C. C. Kao, S. Fisson, G. Vuye, and J. Rivory, "Optical properties of Si nanocrystals embedded in $\mathrm{SiO}_{2}$," Physical Review B, vol. 72, no. 15, Article ID 155319, 2005.

[71] Y. Kanemitsu, Y. Fukunishi, M. Iiboshi, S. Okamoto, and T. Kushida, "Visible luminescence from $\mathrm{Si} / \mathrm{SiO}_{2}$ quantum wells and dots: confinement and localization of excitons," Physica E, vol. 7, no. 3, pp. 456-460, 2000.

[72] M. Cazzanelli, D. Navarro-Urriós, F. Riboli et al., "Optical gain in monodispersed silicon nanocrystals," Journal of Applied Physics, vol. 96, no. 6, pp. 3164-3171, 2004.

[73] M. Ray, K. Jana, N. R. Bandyopadhyay et al., "Blue-violet photoluminescence from colloidal suspension of nanocrystalline silicon in silicon oxide matrix," Solid State Communications, vol. 149, no. 9-10, pp. 352-356, 2009.

[74] S. Gardelis, A. G. Nassiopoulos, N. Vouritzis, and N. Frangis, "Effect of exciton migration on the light emission properties in silicon nanocrystal ensembles," Journal of Applied Physics, vol. 105, Article ID 113509, 2009.

[75] L. Khriachtchev, T. Nikitin, R. Velagapudi, J. Lahtinen, and S. Novikov, "Light-emission mechanism of thermally annealed silicon-rich silicon oxide revisited: what is the role of silicon nanocrystals?" Applied Physics Letters, vol. 94, no. 4, Article ID 043115, 2009.

[76] A. Meldrum, A. Hryciw, A. N. MacDonald et al., "Photoluminescence in the silicon-oxygen system," Journal of Vacuum Science and Technology A, vol. 24, no. 3, pp. 713-717, 2006.

[77] S. Dhara, C. Y. Lu, K. G. M. Nair et al., "Mechanism of bright red emission in Si nanoclusters," Nanotechnology, vol. 19, no. 39, Article ID 395401, 2008.

[78] R. Anthony and U. Kortshagen, "Photoluminescence quantum yields of amorphous and crystalline silicon nanoparticles," Physical Review B, vol. 80, no. 11, Article ID 115407, 2009. 
[79] F. Iacona, G. Franzò, and C. Spinella, "Correlation between luminescence and structural properties of Si nanocrystals," Journal of Applied Physics, vol. 87, no. 3, pp. 1295-1303, 2000.

[80] S. Charvet, R. Madelon, F. Gourbilleau, and R. Rizk, "Spectroscopic ellipsometry analyses of sputtered $\mathrm{Si} / \mathrm{SiO}_{2}$ nanostructures," Journal of Applied Physics, vol. 85, no. 8, pp. 4032-4039, 1999.

[81] S. Takeoka, M. Fujii, and S. Hayashi, "Size-dependent photoluminescence from surface-oxidized Si nanocrystals in a weak confinement regime," Physical Review B, vol. 62, no. 24, pp. 16820-16825, 2000.

[82] Y. Kanemitsu, N. Shimizu, T. Komoda, P. L. F. Hemment, and B. J. Sealy, "Photoluminescent spectrum and dynamics of $\mathrm{Si}^{+}$-ion-implanted and thermally annealed $\mathrm{SiO}_{2}$ glasses," Physical Review B, vol. 54, no. 20, pp. R14329-R14332, 1996.

[83] C. Bonafos, B. Colombeau, A. Altibelli et al., "Kinetic study of group IV nanoparticles ion beam synthesized in $\mathrm{SiO}_{2}$," Nuclear Instruments and Methods in Physics Research, Section $B$, vol. 178, no. 1-4, pp. 17-24, 2001.

[84] M. Zacharias, "Size-controlled Si nanocrystals using $\mathrm{SiO} / \mathrm{SiO}_{2}$ superlattice approach: crystallization, defects and optical properties," in Silicon Nanocrystals, L. Pavesi and R. Turan, Eds., pp. 195-221, Wiley-Vch, 2010.

[85] Z. H. Lu, D. J. Lockwood, and J. M. Baribeau, "Quantum confinement and light emission in $\mathrm{SiO}_{2} / \mathrm{Si}$ superlattices," Nature, vol. 378, no. 6553, pp. 258-260, 1995.

[86] L. Khriachtchev, S. Novikov, and J. Lahtinen, "Thermal annealing of $\mathrm{Si} / \mathrm{SiO}_{2}$ materials: modification of structural and photoluminescence emission properties," Journal of Applied Physics, vol. 92, no. 10, pp. 5856-5862, 2002.

[87] F. Huisken, H. Hofmeister, B. Kohn, M. A. Laguna, and V. Paillard, "Laser production and deposition of light-emitting silicon nanoparticles," Applied Surface Science, vol. 154, pp. 305-313, 2000.

[88] M. Zacharias, J. Bläsing, P. Veit, L. Tsybeskov, K. Hirschman, and P. M. Fauchet, "Thermal crystallization of amorphous $\mathrm{Si} / \mathrm{SiO}_{2}$ superlattices," Applied Physics Letters, vol. 74, no. 18, pp. 2614-2616, 1999.

[89] X. Portier, C. Ternon, F. Gourbilleau, C. Dufour, and R. Rizk, "Anneal temperature dependence of $\mathrm{Si} / \mathrm{SiO}_{2}$ superlattices photoluminescence," Physica E, vol. 16, no. 3-4, pp. 439-444, 2003.

[90] H. Coffin, C. Bonafos, S. Schamm et al., "Oxidation of Si nanocrystals fabricated by ultralow-energy ion implantation in thin $\mathrm{SiO}_{2}$ layers," Journal of Applied Physics, vol. 99, no. 4, Article ID 044302, 2006.

[91] L. Khriachtchev, M. Räsänen, S. Novikov, and L. Pavesi, "Systematic correlation between Raman spectra, photoluminescence intensity, and absorption coefficient of silica layers containing Si nanocrystals," Applied Physics Letters, vol. 85, no. 9, pp. 1511-1513, 2004.

[92] L. Khriachtchev, M. Räsänen, S. Novikov, and J. Lahtinen, "Tunable wavelength-selective waveguiding of photoluminescence in Si-rich silica optical wedges," Journal of Applied Physics, vol. 95, no. 12, pp. 7592-7601, 2004.

[93] S. Novikov, J. Sinkkonen, T. Nikitin, L. Khriachtchev, M. Räsänen, and E. Haimi, "Free-standing $\mathrm{SiO}_{2}$ films containing Si nanocrystals directly suitable for transmission electron microscopy," Microelectronics Journal, vol. 39, no. 3-4, pp. 518-522, 2008.

[94] F. Iacona, C. Bongiorno, C. Spinella, S. Boninelli, and F. Priolo, "Formation and evolution of luminescent $\mathrm{Si}$ nanoclusters produced by thermal annealing of $\mathrm{SiO}_{x}$ films," Journal of Applied Physics, vol. 95, no. 7, pp. 3723-3732, 2004.

[95] L. Tsybeskov, K. D. Hirschman, S. P. Duttagupta et al., "Nanocrystalline-silicon superlattice produced by controlled recrystallization," Applied Physics Letters, vol. 72, no. 1, pp. 43-45, 1998.

[96] E. Werwa, A. A. Seraphin, L. A. Chiu, C. Zhou, and K. D. Kolenbrander, "Synthesis and processing of silicon nanocrystallites using a pulsed laser ablation supersonic expansion method," Applied Physics Letters, vol. 64, no. 14, pp. 1821-1823, 1994.

[97] F. Gourbilleau, C. Ternon, D. Maestre, O. Palais, and C. Dufour, "Silicon-rich $\mathrm{SiO}_{2} / \mathrm{SiO}_{2}$ multilayers: a promising material for the third generation of solar cell," Journal of Applied Physics, vol. 106, no. 1, Article ID 013501, 2009.

[98] S. Boninelli, F. Iacona, G. Franzò, C. Bongiorno, C. Spinella, and F. Priolo, "Formation, evolution and photoluminescence properties of Si nanoclusters," Journal of Physics Condensed Matter, vol. 19, no. 22, Article ID 225003, 2007.

[99] L. Khriachtchev, T. Nikitin, C. J. Oton et al., "Optical properties of silicon nanocrystals in silica: results from spectral filtering effect, $\mathrm{m}$-line technique, and $\mathrm{x}$-ray photoelectron spectroscopy," Journal of Applied Physics, vol. 104, no. 10, Article ID 104316, 2008.

[100] L. Khriachtchev, M. Räsänen, and S. Novikov, "Efficient wavelength-selective optical waveguiding in a silica layer containing Si nanocrystals," Applied Physics Letters, vol. 83, no. 15, pp. 3018-3020, 2003.

[101] L. Khriachtchev, S. Novikov, J. Lahtinen, and M. Räsänen, "Wavelength-selective optical waveguiding of photoluminescence in a thermally annealed $\mathrm{Si} / \mathrm{SiO}_{2}$ superlattice," Journal of Physics Condensed Matter, vol. 16, no. 18, pp. 3219-3228, 2004.

[102] J. Valenta, T. Ostatnický, I. Pelant, R. G. Elliman, J. Linnros, and B. Hönerlage, "Microcavity-like leaky mode emission from a planar optical waveguide made of luminescent silicon nanocrystals," Journal of Applied Physics, vol. 96, no. 9, pp. 5222-5225, 2004.

[103] L. Khriachtchev, D. Navarro-Urrios, L. Pavesi, C. J. Oton, N. E. Capuj, and S. Novikov, "Spectroscopy of silica layers containing Si nanocrystals: experimental evidence of optical birefringence," Journal of Applied Physics, vol. 101, no. 4, Article ID 044310, 2007.

[104] M. K. Miller, K. F. Russell, and G. B. Thompson, "Strategies for fabricating atom probe specimens with a dual beam FIB," Ultramicroscopy, vol. 102, no. 4, pp. 287-298, 2005.

[105] K. Thompson, D. Lawrence, D. J. Larson, J. D. Olson, T. F. Kelly, and B. Gorman, "In situ site-specific specimen preparation for atom probe tomography," Ultramicroscopy, vol. 107, no. 2-3, pp. 131-139, 2007.

[106] D. Blavette, B. Deconihout, A. Bostel, J. M. Sarrau, M. Bouet, and A. Menand, "The tomographic atom probe: a quantitative three-dimensional nanoanalytical instrument on an atomic scale," Review of Scientific Instruments, vol. 64, no. 10, pp. 2911-2919, 1993.

[107] T. F. Kelly and M. K. Miller, "Atom probe tomography," Review of Scientific Instruments, vol. 78, Article ID 031101, 2007.

[108] E. Talbot, R. Lardé, F. Gourbilleau, C. Dufour, and P. Pareige, "Si nanoparticles in $\mathrm{SiO}_{2}$ : An atomic scale observation for optimization of optical devices," Europhysics Letters, vol. 87, Article ID 26004, 2009. 
[109] R. Lardé, E. Talbot, P. Pareige et al., "Evidence of superparamagnetic Co clusters in pulsed laser deposition-grown $\mathrm{Zn}_{0.9} \mathrm{Co}_{0.1} \mathrm{O}$ thin films using atom probe tomography," Journal of the American Chemical Society, vol. 133, no. 5, pp. 1451-1458, 2011.

[110] M. Roussel, E. Talbot, F. Gourbilleau, and P. Pareige, "Atomic characterization of $\mathrm{Si}$ nanoclusters embedded in $\mathrm{SiO}_{2}$ by atom probe tomography," Nanoscale Research Letters, vol. 6, Article ID 164, 2011.

[111] R. J. Temkin, "An analysis of the radial distribution function of $\mathrm{SIO}_{x}$," Journal of Non-Crystalline Solids, vol. 17, no. 2, pp. 215-230, 1975.

[112] H. R. Philipp, "Optical and bonding model for noncrystalline $\mathrm{SiO}_{x}$ and $\mathrm{SiO}_{x} \mathrm{~N}_{y}$ materials," Journal of NonCrystalline Solids, vol. 8-10, no. C, pp. 627-632, 1972.

[113] G. Franzò, M. Miritello, S. Boninelli et al., "Microstructural evolution of $\mathrm{SiO}_{x}$ films and its effect on the luminescence of Si nanoclusters," Journal of Applied Physics, vol. 104, Article ID 094306, 2008.

[114] L. Khriachtchev, M. Räsänen, S. Novikov, O. Kilpelä, and J. Sinkkonen, "Raman scattering from very thin Si layers of $\mathrm{Si} / \mathrm{SiO}_{2}$ superlattices: experimental evidence of structural modification in the $0.8-3.5 \mathrm{~nm}$ thickness region," Journal of Applied Physics, vol. 86, no. 10, pp. 5601-5608, 1999.

[115] L. Khriachtchev, S. Novikov, and O. Kilpelä, "Optics of $\mathrm{Si} / \mathrm{SiO}_{2}$ superlattices: application to Raman scattering and photoluminescence measurements," Journal of Applied Physics, vol. 87, no. 11, pp. 7805-7813, 2000.

[116] I. H. Campbell and P. M. Fauchet, "The effects of microcrystal size and shape on the one phonon Raman spectra of crystalline semiconductors," Solid State Communications, vol. 58, no. 10, pp. 739-741, 1986.

[117] L. Khriachtchev, O. Kilpelä, S. Karirinne, J. Keränen, and T. Lepistö, "Substrate-dependent crystallization and enhancement of visible photoluminescence in thermal annealing of $\mathrm{Si} / \mathrm{SiO}_{2}$ superlattices," Applied Physics Letters, vol. 78, no. 3, pp. 323-325, 2001.

[118] H. Koyama and P. M. Fauchet, "Laser-induced thermal effects on the optical properties of free-standing porous silicon films," Journal of Applied Physics, vol. 87, no. 4, pp. 17881794, 2000.

[119] L. Khriachtchev, M. Räsänen, and S. Novikov, "Free-standing silica film containing Si nanocrystals: photoluminescence, Raman scattering, optical waveguiding, and laser-induced thermal effects," Applied Physics Letters, vol. 86, no. 14, Article ID 141911, 2005.

[120] L. Khriachtchev, M. Räsänen, and S. Novikov, "Continuouswave laser annealing of free-standing $\mathrm{Si} / \mathrm{SiO}_{2}$ superlattice: modification of optical, structural, and light-emitting properties," Journal of Applied Physics, vol. 100, no. 5, Article ID 053502, 2006.

[121] L. Khriachtchev and S. Novikov, "Laser-induced thermal effects on $\mathrm{Si} / \mathrm{SiO}_{2}$ free-standing superlattices," Applied Physics A, vol. 87, no. 4, pp. 761-766, 2007.

[122] J. Macia, E. Martin, A. Perez-Rodriguez et al., "Raman microstructural analysis of silicon-on-insulator formed by high dose oxygen ion implantation: as-implanted structures," Journal of Applied Physics, vol. 82, pp. 3730-3735, 1997.

[123] T. Ujihara, G. Sazaki, K. Fujiwara, N. Usami, and K. Nakajima, "Physical model for the evaluation of solid-liquid interfacial tension in silicon," Journal of Applied Physics, vol. 90, no. 2, pp. 750-755, 2001.
[124] L. Khriachtchev, T. Nikitin, M. Räsänen et al., "Continuouswave laser annealing of Si-rich oxide: a microscopic picture of macroscopic $\mathrm{Si} \mathrm{SiO}_{2}$ phase separation," Journal of Applied Physics, vol. 108, no. 12, Article ID 124301, 2010.

[125] K. Wada, A. Suzuki, H. Sato, and R. Kikuchi, "Soret effect in solids," Journal of Physics and Chemistry of Solids, vol. 46, no. 10, pp. 1195-1205, 1985.

[126] A. Irrera, F. Iacona, I. Crupi et al., "Electroluminescence and transport properties in amorphous silicon nanostructures," Nanotechnology, vol. 17, no. 5, pp. 1428-1436, 2006.

[127] R. J. Walters, G. I. Bourianoff, and H. A. Atwater, "Fieldeffect electroluminescence in silicon nanocrystals," Nature Materials, vol. 4, no. 2, pp. 143-146, 2005.

[128] C. D. Presti, A. Irrera, G. Franzò et al., "Photonic-crystal silicon-nanocluster light-emitting device," Journal of Applied Physics, vol. 88, Article ID 033501, 2006.

[129] Y. Kanemitsu, H. Uto, Y. Masumoto, T. Matsumoto, T. Futagi, and H. Mimura, "Microstructure and optical properties of free-standing porous silicon films: size dependence of absorption spectra in Si nanometer-sized crystallites," Physical Review B, vol. 48, no. 4, pp. 2827-2830, 1993.

[130] F. L. Zhou and J. D. Head, "Role of $\mathrm{Si}=\mathrm{O}$ in the photoluminescence of porous silicon," Journal of Physical Chemistry B, vol. 104, no. 43, pp. 9981-9986, 2000.

[131] S. Godefroo, M. Hayne, M. Jivanescu et al., "Classification and control of the origin of photoluminescence from $\mathrm{Si}$ nanocrystals," Nature Nanotechnology, vol. 3, no. 3, pp. 174$178,2008$.

[132] G. Conibeer, “Third-generation photovoltaics," Materials Today, vol. 10, no. 11, pp. 42-50, 2007.

[133] E. Garnett and P. Yang, "Light trapping in silicon nanowire solar cells," Nano Letters, vol. 10, no. 3, pp. 1082-1087, 2010.

[134] K. Q. Peng, X. Wang, L. Li, X. L. Wu, and S. T. Lee, "Highperformance silicon nanohole solar cells," Journal of the American Chemical Society, vol. 132, no. 20, pp. 6872-6873, 2010.

[135] H. A. Atwater and A. Polman, "Plasmonics for improved photovoltaic devices," Nature Materials, vol. 9, no. 3, pp. 205213, 2010.

[136] S. Pillai, I. Perez-Wurfl, G. J. Conibeer, and M. A. Green, "Surface plasmons for improving the performance of quantum dot structures for third generation solar cell applications," Physica Status Solidi (C) Current Topics in Solid State Physics, vol. 8, no. 1, pp. 181-184, 2011.

[137] Z. Yuan, G. Pucker, A. Marconi et al., "Silicon nanocrystals as a photoluminescence down shifter for solar cells," Solar Energy Materials and Solar Cells, vol. 95, no. 4, pp. 12241227, 2011.

[138] D. Timmerman, J. Valenta, K. Dohnalova, W. D. A. M. de Boer, and T. Gregorkiewicz, "Step-like enhancement of luminescence quantum yield of silicon nanocrystals," Nature Nanotechnology, vol. 6, pp. 710-713, 2011.

[139] D. Di, I. Perez-Wurfl, G. Conibeer, and M. A. Green, "Formation and photoluminescence of Si quantum dots in $\mathrm{SiO}_{2} / \mathrm{Si}_{3} \mathrm{~N}_{4}$ hybrid matrix for all-Si tandem solar cells," Solar Energy Materials and Solar Cells, vol. 94, no. 12, pp. 22382243, 2010.

[140] D. Maestre, O. Palais, D. Barakel et al., "Structural and optoelectrical characterization of $\mathrm{Si}-\mathrm{SiO}_{2} / \mathrm{SiO}_{2}$ multilayers with applications in all Si tandem solar cells," Journal of Applied Physics, vol. 107, Article ID 064321, 2010. 
[141] R. P. Nalini, C. Dufour, J. Cardin, and F. Gourbilleau, "New Si-based multilayers for solar cell applications," Nanoscale Research Letters, vol. 6, no. 1, Article ID 156, 2011.

[142] R. P. Nalini, L. Khomenkova, O. Debieu et al., " $\mathrm{SiO}_{x} / \mathrm{SiN}_{y}$ multilayers for photovoltaic and photonic applications," Nanoscale Research Letters, vol. 7, Article ID 124, 2012.

[143] E. C. Cho, S. Park, X. Hao et al., "Silicon quantum dot/crystalline silicon solar cells," Nanotechnology, vol. 19, no. 24, Article ID 245201, 2008.

[144] T. Nikitin, L. Khriachtchev, M. Räsänen, and S. Novikov, "Optical memory of silicon nanocrystals with submicron spatial resolution and very high thermal stability," Applied Physics Letters, vol. 94, no. 17, Article ID 173116, 2009. 


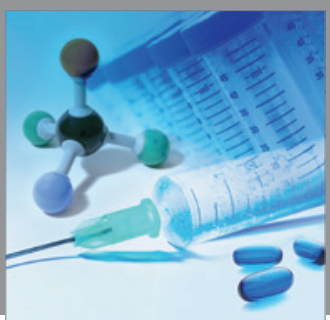

International Journal of

Medicinal Chemistry

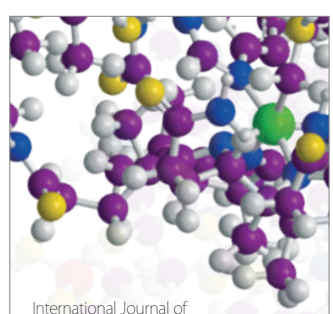

Carbohydrate Chemistry

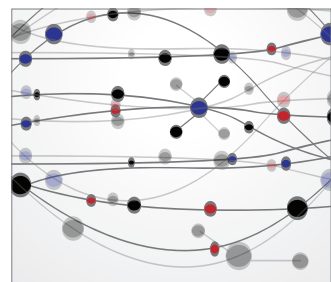

The Scientific World Journal
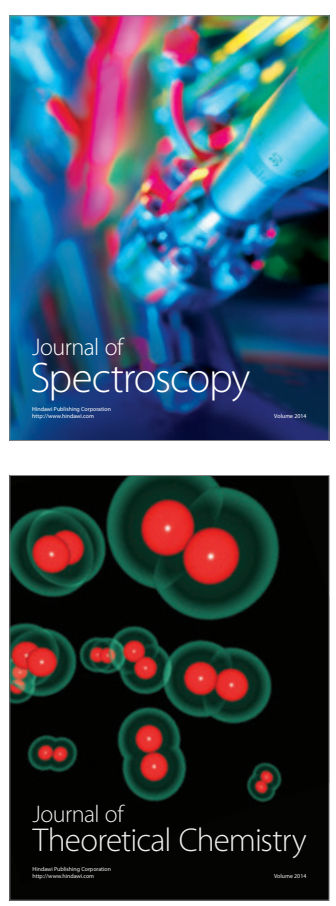
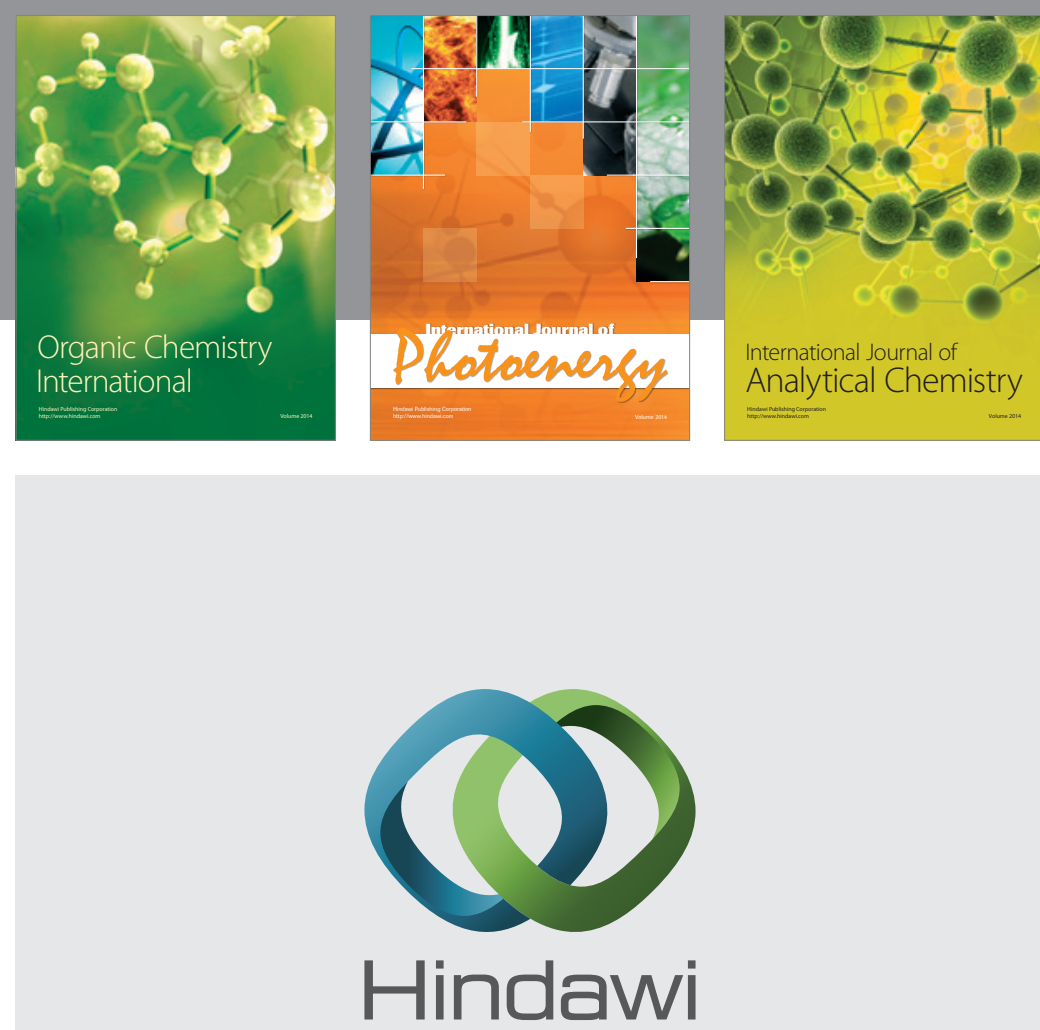

Submit your manuscripts at

http://www.hindawi.com
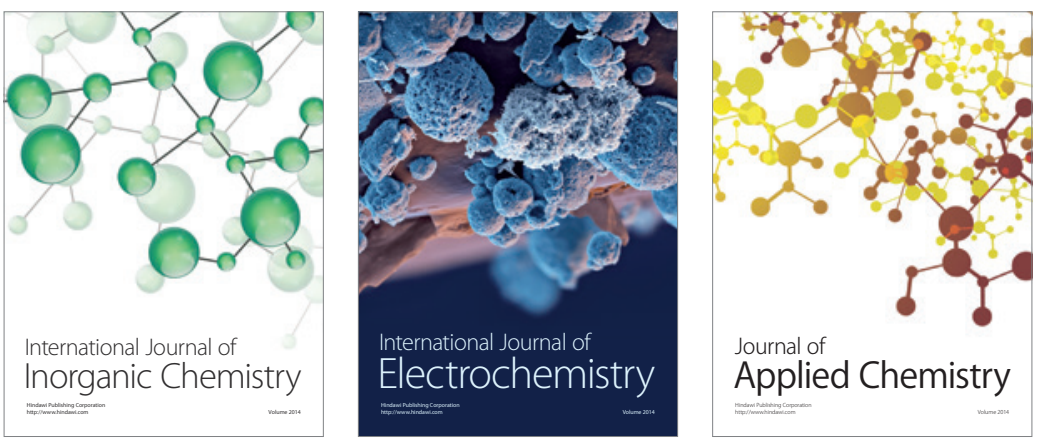

Journal of

Applied Chemistry
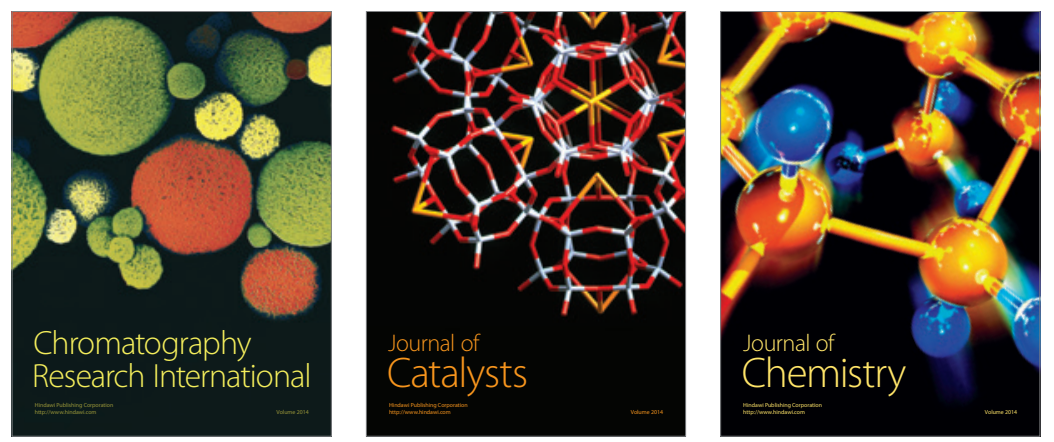
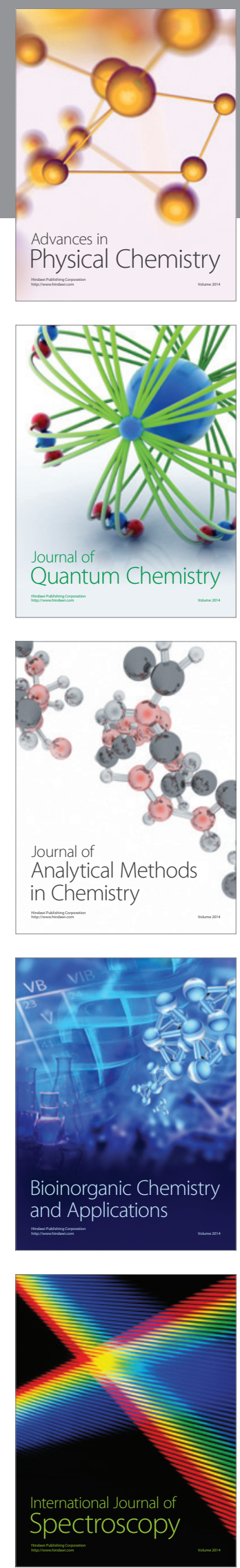